Nouvelles perspectives en sciences sociales

\title{
Quelle sociologie relationnelle? Une perspective non
} relationniste

\section{Which Relational Sociology? A Non-Relationist Perspective}

\section{Pierpaolo Donati}

Volume 13, numéro 1, novembre 2017

URI : https://id.erudit.org/iderudit/1044020ar

DOI : https://doi.org/10.7202/1044020ar

Aller au sommaire du numéro

\section{Éditeur(s)}

Prise de parole

ISSN

1712-8307 (imprimé)

1918-7475 (numérique)

Découvrir la revue

Citer cet article

Donati, P. (2017). Quelle sociologie relationnelle? Une perspective non relationniste. Nouvelles perspectives en sciences sociales, 13(1), 325-371. https://doi.org/10.7202/1044020ar

\section{Résumé de l'article}

Dans cet article, l'auteur présente sa version originale d'une sociologie relationnelle, laquelle repose sur un réalisme critique, qu'on appelle aussi " théorie relationnelle de la société ». Elle partage avec d'autres versions de la sociologie relationnelle l'objectif de comprendre les faits sociaux en tant qu'entités constituées relationnellement. Mais elle diffère des versions constructivistes radicales (ici nommées sociologies relationnistes) par la façon dont les relations sociales sont définies, les voies par lesquelles elles sont générées et elles changent (morphogénèse sociale), et la manière dont elles configurent les formations sociales. L'article met au clair les avantages qu'offre cette perspective originale pour l'explication des phénomènes sociaux émergents. En particulier, montre-t-il, cette perspective peut orienter la recherche sociale vers des réalités invisibles ou immatérielles. Empiriquement, elle peut montrer comment des formes sociales nouvelles sont créées, comment elles changent ou comment elles se détruisent en fonction de divers processus de valorisation ou de dévalorisation des relations sociales. Ultimement, cette approche offre la possibilité de mettre en lumière des processus relationnels qui peuvent permettre aux agents sociaux humains de mieux se réaliser et leur donner, en tant que sujets relationnels, l'occasion d'accéder au bien-vivre. 


\title{
Quelle sociologie relationnelle? Une perspective non relationniste ${ }^{1}$
}

\author{
Pierpaolo Donati \\ Université de Bologne
}

\section{Introduction : le tournant relationnel}

T a sociologie est la science de la société. Mais qu’est-ce que la _société? Qu'est-ce que le social et comment pouvons-nous l'examiner de façon scientifique? Ces questions trouveront une réponse grâce à la théorie relationnelle de la société que je développe depuis 1983. Ma sociologie relationnelle diffère des autres sociologies soi-disant relationnelles (je les appelle relationnistes au lieu de relationnelles, comme je l'expliquerai plus tard). Ces autres théories sont figurationnelles, transactionnelles ou, d'une façon ou d'une autre, réductionnistes. J'invoquerai l'individualisme et l'holisme méthodologiques pour proposer plutôt une méthodologie relationnelle (et non un relationnisme méthodologique).

Selon moi, la sociologie relationnelle est une façon d'observer et de penser qui présume que les problèmes sociaux sont générés par les relations sociales; elle a pour but de comprendre ces problèmes et, si possible, de les régler, non seulement par des actions volontaires ou individuelles, non seulement par des actions collectives ou structurales, mais par de nouvelles relations

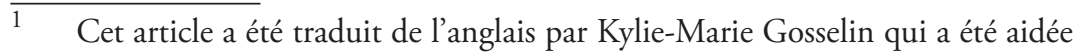
par Benoît Trudel. 
sociales et une nouvelle articulation de ces relations. L'essence même du social est relationnelle : les faits sociaux sont compris et expliqués en présumant que le début de tout fait social est la relation. "Au commencement il y a la relation». Nul ne peut nier la complexité de cette approche qui aspire à avancer une théorie et une méthode qui convient à un ordre de réalité plus complexe ${ }^{2}$.

Ma sociologie relationnelle voit la réalité d'une perspective qui est à la fois spécifique et générale, celle de relationnalité ${ }^{3}$. Elle est descriptive et explicative; bien que pratique, sensible à la normativité, elle ne vise ni les individus ni les structures sociales comme telles, elle s'intéresse plutôt aux relations sociales : elle les analyse, les interprète et leur attribue une valeur selon les problèmes engendrés et les solutions potentielles. Quand j'écris qu'elle est "sensible à la normativité», je ne dis pas que ma sociologie relationnelle est idéologique ou directive; le sociologue doit éviter la confusion entre la recherche scientifique et une quelconque éthique appliquée a priori. Cela veut dire que nous devons étudier la science en interpellant notre conscience, ce qui suppose que la sociologie ne peut être moralement neutre : elle demeure impartiale, certes, mais doit tenir compte de l'orientation des valeurs en jeu, par exemple dans le contexte des droits de la personne ${ }^{4}$.

Pour ce qui est de l'application, orientée vers l'intervention en réseau, il est question de produire un changement permettant

$2 \quad$ En ce qui concerne la définition des concepts d'approche, de paradigme, de théorie et de méthode, je renvoie le lecteur au chapitre 1 de Pierpaolo Donati, Introduzione alla sociologia relazionale (Milan, Franco Angeli, 1986 [1983]). On lira aussi des mises au point sur ces concepts et sur leurs relations dans Pierpaolo Donati, Relational Sociology. A New Paradigm for the Social Sciences (Londres et New York, Routledge, 2011).

3 La perspective est exhaustive dans la mesure où les relations sociales sont répandues dans toute la société et où la société est constituée de relations sociales, même si nous percevons ces dernières, de temps à autres, comme étant économiques, politiques, juridiques, psychologiques, etc. La perspective est également spécifique dans la mesure où la relation n'est pas perçue d'un angle logique, économique, juridique ou politique, mais plutôt d'un angle social, ce qui signifie que les sujets mutuellement impliqués lui inculquent un sens.

4 Elżbieta Halas, "Through Social Values to the Reinterpretation of Sociology's Ethical Neutrality ", Sociologia e Politiche Sociali, vol. 19, n ${ }^{\circ}$ 1, 2016, p. 67-79. 
aux sujets de gérer leurs propres relations déterminantes, qu'elles soient actuelles ou potentielles. La méthode utilisée amène le sujet à exploiter ses ressources humaines et matérielles, évidentes ou latentes, pour atteindre un niveau adéquat d'autorégulation - ou minimalement un niveau permettant de régler des problèmes qui seraient autrement perçus et classifiés comme des problèmes d'acteurs individuels ou d'entités collectives abstraites.

La sociologie relationnelle n'est pas inventée de toute pièce ou déterminée a priori par une théorie fermée ou autoréférentielle. Historiquement, elle présuppose l'émergence d'une nouvelle forme de société que je qualifie de relationnelle ${ }^{5}$. De par sa nature même, cette sociologie est une conséquence de la mondialisation. Elle a comme principe directeur la génération continuelle des relations sociales dans le cadre des processus de différenciation, de conflit et d'intégration, tant sur le plan intersubjectif (dans les réseaux primaires) que général (dans les réseaux secondaires, interpersonnels et organisationnels). Ce genre de société exige une vision théorique et appliquée de la réalité sociale qui est suffisamment ouverte à elle-même et par rapport à elle-même pour permettre de penser de façon relationnelle dans un mode réflexif 6 .

Je vous présente ici un sommaire de cette approche pour vous convaincre que seule une théorie avec ses propres principes, à la fois spécifiques et largement applicables, peut fournir une compréhension et une application pragmatique nécessaires pour donner à la sociologie son caractère pratique.

La difficulté principale est de distinguer cette approche de celles qui se disent relationnelles, qui accentuent le rôle des relations sociales, mais qui, en fait, ont une compréhension réductionniste de ces relations. Je pense surtout à plusieurs

5 Pierpaolo Donati, Relational Sociology. A New Paradigm for the Social Sciences, op. cit., p. 56-58.

6 Sur le sujet de la réflexivité, voir Margaret S. Archer (dir.), Conversations on Reflexivity (Londres, Routledge, 2010) et Margaret S. Archer, The Reflexive Imperative in Late Modernity (Cambridge, Cambridge University Press, 2012). 
versions du fonctionnalisme (de Talcott Parsons ${ }^{7}$ à Niklas Luhmann $^{8}$ ), à la plupart des conceptions structuralistes des relations sociales (Ronald $S$. Burt ${ }^{9}$, Barry Wellman ${ }^{10}$, etc.) et à la lecture relativiste de ces relations telle que proposée par des sociologues néo-pragmatistes (Mustafa Emirbayer ${ }^{11}$, François Dépelteau $^{12}$, Christopher Powell ${ }^{13}$ ) qui proposent une sociologie plutôt transactionnelle que relationnelle.

Mon approche se fonde sur un type de réalisme que j'appelle réalisme relationnel ${ }^{14}$ et qui est à la fois analytique, critique et relationnel. Ce réalisme pourrait remplacer les approches relationnelles qui se basent sur une ontologie constructiviste, sans vouloir unifier toutes les approches sociologiques autour de la notion de la relation (au détriment des notions de système ou de réseau). La critique de Rachid Bagaoui selon laquelle j'aurais proposé une théorie unifiante pour remplacer toutes les autres ${ }^{15}$ est trompeuse : ma sociologie relationnelle est conçue, depuis le début, comme un cadre général pour lier le meilleur de chaque

Talcott Parsons, The Social System, New York, Free Press, 1951.

Niklas Luhmann, Social Systems, Stanford, Stanford University Press, 1995.

Voir par exemple, Ronald S. Burt, Toward a Structural Theory of Action. Network Models of Social Structure, Perception, and Action (New York, Academic Press, 1982), et Structural Holes. The Social Structure of Competition, (Cambridge (MA), Harvard University Press, 1992).

Voir par exemple, Barry Wellman, «The Community Question: The Intimate Networks of East Yorkers ", American Journal of Socioogy, vol. 84, n 5, 1979, p. 1201-1231, et "Structure Analysis: From Method and Metaphor to Theory and Substance ", dans Barry Wellman et S. D. Berkowitz (dir.), Social Structures: A Network Approach, Cambridge, Cambridge Univesity Press, 1988, p. 19-61.

Mustafa Emirbayer, "Manifesto for a Relational Sociology ", American Journal of Sociology, vol. 103, n ${ }^{\circ}$ 2, 1997, p. 281-317.

François Dépelteau, "Relational Sociology, Pragmatism, Transactions, and Social Field ", International Review of Sociology, vol. 25, n 1, 2015, p. 45-64. Christopher Powell, " Radical Relationism: A Proposal ", dans François Dépelteau et Christopher Powell (dir.), Conceptualizing relational Sociology: Ontological and Theoretical Issues, New York, Palgrave, p. 187-207.

Pierpalo Donati, Introduzione alla sociologia relazionale, op. cit., 1983 [puis 1986], p. 10, et Relational Sociology. A new Paradigm for the Social, op. cit., p. 97-119.

Rachid Bagaoui, "Un paradigme systémique relationnel est-il possible? Proposition d'une typologie relationnelle ", Nouvelles perspectives en sciences sociales, vol. 3, $\mathrm{n}^{\circ} 1$, 2007, p. 151-175. 
sociologie ${ }^{16}$. Pour éviter ce type de théorie, Bagaoui propose une sociologie relationnelle plurale ${ }^{17}$. Certes, il faut éviter une théorie unifiante, qui serait constrictive et restrictive, mais je ne vois pas l'utilité de l'adjectif "plurale»; si la théorie est réellement relationnelle, elle est nécessairement pluraliste, tant et aussi longtemps qu' elle puisse traiter du caractère essentiel de la relation, c'est-à-dire la valorisation à la fois des points communs et des différences, ce que j'appelle l'énigme de la relation ${ }^{18}$. C'est précisément l'absence ou le rejet de la relation qui mine le pluralisme.

À mon sens, pour avoir une approche relationnelle, il faut faire de la relation sociale l'unité de base de l'analyse. Il ne s'agit pas de remplacer le concept d'individu ou de système par celui de relation. Au contraire, adopter la relation comme unité de base permet de mieux élucider les concepts d'individu et de système d'un point de vue sociologique. La sociologie devrait observer le fait social, le penser et intervenir sur lui, avec la reconnaissance fondamentale qu'il est - ce même fait social - l'expression de la relationnalité générée par les humains; il est l'expression ni d'un système (à l'instar de l'holisme méthodologique) ni d'une action individuelle (à l'instar de l'individualisme méthodologique). Comme je l'explique plus loin, mon approche garde la pertinence de la perspective humaine d'une manière précise, c'est-à-dire en admettant que la société n'est pas constituée d'êtres humains, mais que ces derniers la créent; elle est formée de relations ${ }^{19}$.

\footnotetext{
16 Pierpaolo Donati, Introduzione alla sociologia relazionale, op. cit., 1983 [puis 1986], p. 11-12.

17 Rachid Bagaoui, op. cit., p. 173.

18 Pierpaolo Donati, L'enigma della relazione, Milan-Udine, Mimesis edizioni, 2015.

19 Autrement dit, la société maintient une préoccupation humaniste, mais qui n'est plus comprise dans des termes classiques (la coïncidence du social et de l'humain qui fait en sorte que le social est compris d'abord comme étant humain), mais plutôt comme un processus émergent de la différenciation de l'humain et du social [voir Pierpaolo Donati, La società dell'umano (GenovaMilano, Marietti, 2009) et "What Does a "Good Life" Mean in a Morphogenic Society? The Viewpoint of Relational Sociology " (dans Margaret S. Archer (dir.), Morphogenesis and Human Flourishing, Dordrecht, Springer, 2017, p. 137-161)].
} 
Admettre que la relation sociale constitue sa propre réalité nous permet de la distinguer de la théorie des systèmes et de la théorie de l'action, et ce, sans prendre position ni pour l'une ni pour l'autre. Cela nous permet surtout de voir dans les relations sociales une réalité qui, bien qu'invisible, inexpliquée, et souvent incertaine, constitue le substratum sur lequel la société est construite et évolue, à la fois dans ses origines et dans sa quête de solutions humaines aux problèmes sociaux; voilà une approche différente de celle que propose le constructionnisme. Je m’oppose avant tout à ces sociologies "relationnelles» qui réduisent les relations sociales à des communications pures et qui croient que les relations sociales peuvent être construites de diverses manières (comme le mentionne Luhmann ${ }^{20}$ ). À mon avis, si les relations sont contingentes, ce n'est pas vrai qu'elles peuvent toujours être «autrement», comme le voudraient les relationnistes.

Bref, la sociologie relationnelle se fonde sur le tournant relationnel de la société que provoque la modernité et dépasse ce tournant. Elle fait avancer la vision relationnelle qui est d'abord explorée et interprétée par Karl Marx, Max Weber et Georg Simmel, et propose une théorie relationnelle intégrale qui est articulée dans une ontologie, une épistémologie et une méthodologie sui generis et qui se solde en une pratique sociale spécifique.

\section{Les prémisses ontologiques de la pensée relationnelle}

L'émergence de ce que j'appelle la sociologie relationnelle est un processus historique qui produit un changement de paradigme du simple au complexe et qui se fait le reflet de ce changement. Ce processus est une transformation radicale des statuts ontologique, épistémologique et phénoménologique des relations sociales, autant dans les sciences que dans la société. Pour comprendre cette transformation, nous devons nous munir d'une approche qui, nous permet, d'une part, de saisir la réalité des relations sociales dans leurs propres termes et, d'autre part, de préciser que ce sont ces relations sociales qui constituent l'objet

$\overline{20}$ Niklas Luhmann, op. cit. 
d'analyses et d'interventions. Nous entrons ici dans le domaine de la pensée relationnelle, que nous discuterons en détail.

a) Les relations sociales sont réelles selon leurs propres termes

Dire que les relations sociales ont leur propre réalité signifie qu'elles ne sont pas simplement dérivées d'autre chose. La dérivation est un processus complexe qui génère un fait émergent avec sa propre existence. Il s'agit d'une réalité en bonne et due forme qui est composée de strates internes, chacune desquelles demande une attention toute particulière, ainsi qu'un traitement théorique et pratique. Cet ordre ne peut être réduit à un seul facteur ou à une variable sociale unique (comme le pouvoir ou l'utilité économique). Une personne ne peut pas vivre sans relations sociales, tout comme elle ne peut pas vivre sans oxygène, car c'est la relationnalité qui est sociale. Les relations sociales sont constitutives pour un humain, tout comme l'oxygène l'est pour son corps, mais l'être humain n'est réductible ni aux unes ni à l'autre. L'interruption des relations avec autrui représente l'interruption de la relation avec soi-même : voilà ce à quoi les sciences sociales s'intéressent.

On peut situer l'observation sociologique à deux niveaux :

- le niveau de base : les relations sont observées, mais elles sont aussi analysées en misant sur un facteur ou une variable (un moyen d'échange généralisé, par exemple, comme l'argent ou le pouvoir) qui va, dans les échanges sociaux, du point $A$ au point $B$ et vice versa;

- le niveau réflexif : on observe non pas les facteurs au sein de la relation, mais plutôt la réalité même des relations, sa structure dynamique. En fait, les relations sont autonomes dès qu'elles existent : les entités concrètes comme les événements ou les institutions historiques de la société peuvent être observées et interprétées à titre de réseaux relationnels qui découlent d'un contexte dynamique et concurrentiel de relations sociales. 
Certes, les relations sociales ne peuvent pas traverser la rue. Nous savons cependant qu'elles existent, non seulement parce qu'elles se matérialisent en formes, en institutions et en mouvements sociaux, mais aussi parce qu'on en fait l'expérience. Admettre qu'elles sont caractérisées par l'éventualité ne veut pas dire qu'elles ne sont pas réelles. La couleur de nos yeux, par exemple, est une caractéristique éventuelle, mais elle est partie intégrante de la réalité.

Quelle est donc cette réalité? Elle est celle d'une relation entre nous et les choses, entre nous-mêmes et les autres; elle n'est pas simplement logique ou psychique. La relation sociale se distingue des relations logiques et psychiques de trois façons :

- elle se réfère à quelque chose par voie de symboles (refero),

- elle se connecte structurellement (religo),

- elle émerge du radical de l'acte réciproque (relazione en italien) qu'est l'action mutuelle. ${ }^{21}$

Pour étudier les relations sociales, le chercheur doit tenir compte du fait qu'il est lui-même une tierce partie ${ }^{22}$ et qu'il doit choisir une méthodologie pertinente, ce que j'appelle une analyse

$\overline{21 \quad C e t t e ~ d e ́ f i n i t i o n ~ d u ~ t e r m e ~ " W e c h s e l w i r k u n g " ~(e f f e t ~ d e ~ r e ́ c i p r o c i t e ́) ~ e s t ~ m i s e ~ d e ~}$ l'avant par Georg Simmel (voir, entre autres, "Comment les formes sociales se maintiennent ", L'année sociologique, $1^{\text {ère }}$ année, 1896-1897, p. 71-109; le texte est publié en français sous la direction d'Émile Durkheim; il est reproduit dans Georg Simmel, Sociologie et épistémologie, Paris, Presses universitaires de France, 1981).

Pour avoir une meilleure idée de la relation dans le contexte de la théorie d'observation autoréférentielle, il faut voir du côté de Hans von Foerster : «Selon le principe de relativité qui rejette une hypothèse lorsqu'elle ne supporte pas simultanément deux propositions (par exemple, les habitants de la Terre et de Vénus peuvent bien déclarer, chacun de leur côté, être le centre de l'univers, mais leurs déclarations sont démenties lorsqu'elles sont confrontées l'une à l'autre), l'affirmation solipsiste s'effondre au moment où je trouve un autre organisme autonome au-delà de moi-même. Ainsi, si le principe de relativité n'est ni une nécessité logique, ni un principe qu'on peut dire vrai ou faux, le point crucial est que je suis libre de choisir si j'accepte ou non ce principe. Si je le rejette, je suis le centre de l'univers, mes rêves et cauchemars sont ma réalité, mon langage est un monologue et ma logique est monologique. Si je l'adopte, personne n'est le centre de l'univers. Comme dans un univers héliocentrique, il doit y avoir un troisième élément qui est le point de référence essentiel : c'est la relation toi et moi et cette relation (la réalité 
relationnelle $e^{23}$. Le social est une matière relationnelle, non une projection d'individus ou une entité holistique qui existe entre les acteurs, entre l'observé et l'observateur. En contraste avec la sphère physique, où les relations entre les entités matérielles sont mécaniques, elles sont communicatives et interprétatives dans la sphère sociale. La rétroaction s'avère alors relationnelle, et non mécanique ${ }^{24}$.

Ainsi, en adoptant la perspective relationnelle, l'observateur devrait se situer à un niveau invisible, mais bien réel, selon lequel la relation devient un troisième élément. Il doit toujours être situé ainsi s'il veut empêcher la relativité épistémique de se transformer en relativisme. Bien que ce puisse être difficile à entrevoir, il demeure que la relationnalité existe non seulement au niveau social, mais aussi à l'intersection des autres niveaux de la réalité (biologique, psychique, éthique, politique et économique).

b) La relation sociale définit l'objet de la recherche sociologique

Malgré tout, la relation n'est pas seulement un concept logique et un moyen d'acquérir des connaissances; elle s'avère un point de vue à adopter pour toute personne qui doit faire une analyse sociologique, une interprétation de données ou une réflexion sur les problèmes sociaux pratiques, lequel point de vue lui permettra de bien définir son objet. En effet, pour bien saisir la nature sociale du phénomène, chaque objet doit être défini dans des termes relationnels. J'ai tendance à m'opposer à l'idée selon laquelle la sociologie examine les relations entre les faits sociaux; je crois qu' elle étudie plutôt les faits sociaux comme des relations. La société ne contient pas de relations; elle constitue ces relations.

sociale) = communauté» (Observing Systems, Seaside (CA), Intersystems Publications, 1984, p. 307-308).

23 Pierpaolo Donati, "L'analisi Relazionale: Regole, Quadro Metodologico, Esempi ", dans Pierpaolo Donati (dir.), Sociologia. Una Introduzione allo Studio della Società, Padova, Cedam, 2006, p. 213-227.

24 Pierpaolo Donati, «Morphogenesis and Social Networks: Relational Steering not Mechanical Feedback ", dans Margaret S. Archer (dir.), Social Morphogenesis. Dordrecht, Springer, 2013, p. 205-231. 
C'est dire que les objets de la sociologie, sans compter ses concepts, doivent tous être redéfinis comme des relations.

Au commencement d'un projet de recherche, lorsque le problème est posé (comment et pourquoi X a-t-il lieu? X étant un phénomène sans preuve ni explication intuitive), il faut se rappeler que l'objet d'étude qui découle d'une situation (le phénomène $\mathrm{X}$ ) fait partie d'un contexte relationnel et donne naissance à un deuxième contexte relationnel. L'erreur majeure de l'approche phénoménologique de Husserl est de penser que les relations sociales sont synonymes d'intersubjectivité (l'expression d'empathie, par exemple, ou le partage de valeurs). Nous distinguons le point de vue réaliste critique de celui qui est phénoménologique et qui voit les relations sociales comme une chose venant après coup, émanant des opérations de la conscience transcendantale ou de l'ego. L'idée husserlienne selon laquelle il faut mettre la relation sociale entre parenthèses (une procédure nommée epochè) pour ensuite la voir comme une expression de l'ego transcendantal est autodestructrice ${ }^{25}$. Au contraire, je crois que la sociologie relationnelle devrait maintenir l'affirmation selon laquelle la relation est le point de départ de l'analyse sociale ("au commencement était la relation») et la clé de la connaissance des sujets et des objets, non le contraire. J'ai tenté de trouver des exemples de cette procédure dans des domaines divers comme l'éducation, le capital social, la santé, la famille, la maladie chronique, la citoyenneté, l'État providence, la politique sociale ${ }^{26}$.

c) La relationnalité dans le domaine social nécessite son propre code symbolique

Pour bien comprendre et expliquer la réalité sociale, il est important d'éclaircir le code symbolique utilisé, eu égard au type et au degré de relationnalité nécessaires. Par exemple, je maintiens que les

$\overline{25}$ René Toulemont, L'essence de la société selon Husserl, Paris, Presses universitaires de France, 1962.

26 Pour un aperçu de ces recherches empiriques, voir Paolo Terenzi, Lucia Boccacin et Ricardo Prandini Riccardo (dir.), Lessico di sociologia relazionale, Bologna, il Mulino, 2016. 
codes binaires (oui/non, $0 / 1$, dedans/dehors, etc.) présentent le type et le degré de relationnalité le plus simple.

Le code binaire de Luhmann, s'il est parfois utile, est seulement capable d'appréhender la relationnalité des systèmes interactifs complexes de manière superficielle. En fait, le code binaire est dérivé de la primauté du sous-système économique (muni de son propre code fonctionnel d'efficacité) et de la logique de la compétition, une forme relationnelle qui n'est pas, au fond, interactive. Cela explique que la sociologie de Luhmann ne soit pas utile pour composer avec des relations sociales concrètes. En réalité, le code binaire est valide uniquement pour certains phénomènes autres que sociologiques (logiques, biologiques ou psychiques). Par exemple, nous ne pouvons pas nous en servir pour traiter de la phénoménologie des relations entre le public et le privé sans introduire des simplifications qui ne sont pas appropriées à l'objet d'étude. Dans tous les cas, ce type de réductionnisme est incompatible avec un domaine qui ne peut pas s'occuper de tous les phénomènes sociaux comme s'ils étaient des fonctions binaires - plusieurs ne le sont pas.

Pour pouvoir définir un objet dans des termes relationnels, la sociologie a besoin de codes plus complexes que le système binaire. En effet, des codes et des modèles d'analyse symboliques appropriés sont requis. Un code symbolique doit tenir compte de plus que le relata (ce qui est relié); il doit se pencher sur les relations mêmes, puisque les médiations ne sont pas réductibles à leurs composantes. Des études empiriques sur la relation l'ont bien démontré. Cette dernière est composée de divers apports : l'effet de l'ego sur l'alter (les éléments amenés de l'ego vers l'alter), l'effet de l'alter sur l'ego (la réactivité de l'alter envers l'ego) et l'effet de leur interaction (la combinaison des éléments amenés de l'alter ego et le fonctionnement dans la dynamique de la structure relationnelle $)^{27}$. Ces effets peuvent être observés et

$\overline{27}$ Voir Tony Tam, « Demarcating the Boundaries between Self and the Social: The Anatomy of Centrality in Social Networks " (Social Networks, vol. 11, $\mathrm{n}^{\circ}$ 4, 1989, p. 387-401), et William L. Cook et Albert Dreyer, "The Social Relations Models " (Journal of Marriage and Family, vol. 46, n 3, 1984, p. 679-687). 
mesurés par voie de méthodes convenables : les deux premiers sont analysés au niveau individuel, tandis que le troisième est uniquement observé en adoptant la relation comme unité d'analyse. Comme le veut Simon Laflamme, «la sociologie a pour motif, et non pour objet, les individus ${ }^{28}$ ". Par contre, si nous effectuons l'analyse sociologique selon la théorie du système (tout système faisant partie d'un système plus large), le niveau d'analyse choisi sera toujours incomplet ${ }^{29}$. Tout système doit être défini selon le système qui lui est supérieur, mais le système suprême, de dernier recours, n'existe pas. De cette façon, l'autonomie de chaque acteur et de chaque agent, toujours relative (elle est constituée d'un nœud de relations en lien avec d'autres autonomies dans un contexte), est dissoute. Comme Richard Rorty l'écrit : "Tout ce qui est présenté comme un terme de la relation peut être dissous dans un ensemble de relations, et ainsi de suite pour toujours ${ }^{30} »$.

d) Les relations sociales se fondent sur des systèmes de réseaux qui n'éliminent ni la subjectivité, ni l'importance des éléments individuels, même s'ils transforment ces derniers

Des modèles de réseaux sont requis pour l'analyse et l'intervention sociales qui témoignent à la fois de la contribution des composantes individuelles dans les interactions et des effets qui en résultent. Ainsi, les systèmes sociaux apparaissent comme des condensés de réseaux sociaux ${ }^{31}$.

Cette étude empirique des relations nous permet de distinguer la contribution des sujets individuels de leur conditionnement social. Ici, Tony Tam affirmerait que l'interdépendance n'est pas une idée circulaire ${ }^{32}$. Si l'importance centrale des éléments dans

28 Simon Laflamme, "Sciences sociales et approche relationnelle ", Nouvelles perspectives en sciences sociales, vol. 5, $\mathrm{n}^{\circ} 1,2009$, p. 79-85.

29 Niklas Luhmann, Social Systems, op. cit.

30 Richard Rorty, Philosophy and Social Hope, Londres, Penguin Books, 1999, p. 54 .

31 Pierpaolo Donati, Teoria relazione della società, Milan, Franco Angeli, 1991, chapitre 2.

32 Tony Tam, op. cit. 
un réseau social dépend de leur interdépendance mutuelle, comment pouvons-nous affirmer qu'il y a une part qui est générée de façon autonome par un élément individuel et qui est liée aux caractéristiques de cet élément plutôt qu'à la relation avec autrui? La réponse est que même si nous acceptons une vision du monde dans laquelle chaque personne dépend des autres, nous pouvons tout de même séparer la composante autogénérée de l'élément de celle qui est dérivée d'autrui. Autrement dit, la limite entre un ego et son contexte social est dessinée précisément, même dans un système social. La sociologie relationnelle ne postule pas que le Soi se perd dans l'interdépendance sociale.

La logique des réseaux n'est donc basée ni sur la négation du sujet ni sur la logique circulaire de la phénoménologie. Elle se fonde plutôt sur l'observation, la description et la définition de l'identité de tout acteur social en considérant sa subjectivité et en évitant une circularité indéterminée, laquelle ne peut que se perpétuer ad infinitum. De la perspective de l'ontologie sociale, on dirait que la substance (nature, structure) et la relation (relationnalité) sont des co-principes de la réalité sociale. Les réalistes critiques, eux, diraient que "c'est la nature des choses qui détermine leurs possibilités cognitives pour nous ${ }^{33}$ ». Par conséquent, ce sont les objets à l'étude et nos questions de recherche qui déterminent et expliquent la méthode à suivre.

Selon Hubert Buch-Hansen, il existe une tension dans l'analyse des réseaux sociaux entre les études qui privilégient les méthodes et l'application et celles qui misent sur le caractère social théorique des réseaux. Le premier type, dans plusieurs cas, expose des tendances positivistes, alors que le second comporte un nombre de suppositions directement liées aux principales tendances des réalistes critiques quant à la nature de la réalité et des connaissances sociales ${ }^{34}$. Je suis d'accord que l'analyse des réseaux sociaux peut se détacher de la science sociale positiviste et constituer un instrument valable dans la boîte à outils d'un réaliste critique.

33 Roy Bhaskar, The possibility of naturalism: A philosophical critique of the contemporary human sciences, Brighton, The Harvester Press, 1979, p. 31.

34 Hubert Buch-Hansen, "Social Network Analysis and Critical Realism ", Journal for the Theory of Social Behaviour, vol. 44, n 3, 2013, p. 306-325. 
e) La relationnalité n'est pas relative, mais une détermination spécifique

Les sciences sociales contemporaines sont largement relativistes et envisagent les relations sociales comme un moyen de dissoudre la nature singulière et l'importance tant de la conscience que du phénomène social. L'approche relationnelle, elle, est un moyen de se distancier du relativisme. Au mieux, les approches sociologiques relativistes, qui dominent actuellement, s'approchent d'une position non relativiste dans l'un ou l'autre des cas suivants : soit il existe un ensemble de valeurs empiriquement vérifiable qui a été culturellement transmis et qui limite les possibilités, soit il existe des normes établies de manière interactive qui génèrent une rationalité procédurale, laquelle donne lieu à une autorégulation. L'autorégulation est généralement perçue comme un produit d'Eigenvalues (valeurs comme résultat de la réitération de la communication) ou de dérives naturelles ${ }^{35}$. Le deuxième cas l'emporte sur le premier dès que la persistance (ou la reproduction) des valeurs est envisagée comme un simple prolongement d'orientations culturelles arriérées.

Toutefois, ces deux solutions laissent à désirer, car elles sont très peu sociologiques et relationnelles : la première fait appel à des valeurs imposées (ce qui rappelle la contrainte sociale d'Émile Durkheim) et la seconde se limite à la notion des origines spontanées. La première ne peut que se pencher sur le concept des traditions culturelles, ce qui implique des déficiences qui limitent sérieusement sa force explicative. La seconde réduit la normativité à un phénomène interactif purement évolutif. Dans les deux cas, nul ne sait comment éviter le recours à la normativité plutôt qu'aux solutions aléatoires.

35 Voir Arantza Etxeberria, «Autopoiesis and Natural Drift: Genetic Information, Reproduction, and Evolution Revisited " (Artificial Life, $\mathrm{n}^{\circ}$ 10, 2004, p. 347 360) pour la théorie des dérives naturelles, avancée par Humberto R. Maturana et Francisco J. Varela (The Tree of Knowledge. The Biological Roots of Human Understanding, édition révisée, Boston, Shambhala Publications, 1987). 
Il est possible d'éviter les pièges du réductionnisme et de l'amalgame en ayant recours au schéma pluridimensionnel, comme le fait Jeffrey C. Alexander ${ }^{36}$. Cependant, la pluridimensionnalité n'est pas une approche adéquate pour résoudre les cas d'indétermination qui apparaissent lorsque l'analyse sociologique fait face à des problèmes d'origines et d'uniformité des valeurs standards (ou des symboles, comme l'indique Alexander), lesquels entraîneraient des infractions à la circularité de la multiplicité des variables incluses dans les processus sociaux. Les institutions sociales seraient uniquement le résultat de la culture et des produits de cette culture, et ce, par le biais d'une myriade d'opérations répétées et d'interactions réitérées. Ceux qui ont rejeté ce type de formulation relativiste ont fait appel à des lois structurelles, comme le faisait en grande partie la sociologie du XIX ${ }^{\text {e }}$ siècle. Cependant, la même société moderne et contemporaine cherche à les nier.

Où trouver alors la fracture de cette circularité? Serait-elle dans la structure d'une réalité présupposée a priori? À mes yeux, même cette réponse est sociologiquement non plausible : ce qui fait d'une institution sociale (ou une relation sociale) ce qu'elle est, ce n'est pas qu'elle s'encastre dans une structure prétendument préexistante à l'instar de la représentation symbolique.

À mon avis, la réponse est à la fois simple et complexe : la fracture est enracinée dans les conditions de la relation ellemême, dans la mesure où cette dernière est actualisée par les sujets qui l'ont instituée comme relation déterminante dotée de sa propre structure. Je ne prétends pas que la relation est produite par la seule intention des agents ou qu'elle est le produit d'opérations mécaniques. Je pense plutôt que la détermination de la structure relationnelle est le résultat de facteurs objectifs et subjectifs combinés qui ne correspondent ni aux facteurs objectifs ni aux facteurs subjectifs. C'est une création qui répond à l'énigme de la relation, laquelle consiste à unifier, mais aussi à

36 Jeffrey C. Alexander, Twenty Lectures. Sociological Theory Since World War II, New York, Columbia University Press, 1987. 
différencier ses termes ${ }^{37}$. La relation entre un employeur et son employé, ou entre un docteur et son patient, n'est pas la même que celle d'un couple par exemple. En réponse à la question, il y a un espace réduit pour l'indétermination et les allers-retours dans la chaîne causale s'avèrent limités. La circularité est brisée par la relation elle-même quand elle est prise pour ce qu'elle est : une relation et rien d'autre. La circularité est brisée lorsque la relation est redéfinie selon son propre caractère distinctif, c'està-dire le devoir d'unir deux termes dans un cadre défini. La relation est l'accomplissement de la tâche, celle de créer un Nous-relation (We-relation) entre différents acteurs ou agents sociaux les unissant dans un sujet relationnel qui respecte et favorise leurs différences.

\section{Un exemple}

Pour clarifier les notions ci-dessus, prenons le problème de la famille et de son statut comme réalité sociale.

Dans un sens, la sociologie s'est perdue, soit en adoptant plusieurs analogies (spatiale, biologique, organique, cybernétique, informatique, etc.), soit en voyant la famille comme un endroit, une niche, une cellule ou un système autorégulé. Aujourd'hui, nous ne semblons plus savoir ce qu'est une famille. Il est tentant d'y voir une sous-catégorie d'un groupe primaire, même s'il existe des recherches théoriques ou empiriques qui ont prouvé, depuis longtemps, que la famille n'est ni une forme générique de cohabitation humaine ni un groupe primaire privé de toute différenciation.

D'une perspective relationnelle, si la famille n'était qu'un arrangement communicatif qui permet «l'orientation de la personne vers l'intégralité de sa personne», comme le prétend Luhmann $^{38}$ (1988, p. 75-76), nous nous demandons pourquoi c'est le cas et pourquoi c'est le seul système social, prétendument une interaction pure, qui a cette fonction. Nous avons besoin

\footnotetext{
37 Pierpaolo Donati, L'enigma della relazione, op. cit.

38 Niklas Luhmann, "Sozialsystem Familie ", dans System Familie, 1, 1988, p. 75-76.
} 
d'un raisonnement plus détaillé. Si la famille est ainsi, c'est qu’à titre de relation sociale précise elle détient une structure sui generis et un code symbolique propre qui lui permet d'assurer certaines relations entre les sexes et les générations. Évidemment, je ne doute pas que la famille permette une orientation personnelle ou communicationnelle de l'individu comme personne (un individu dans une relation et non comme un atome par exemple). Mais il faut faire attention lorsqu'on prétend que cette fonction est réservée à la famille, et que cette dernière n'a que cette fonction. De manière générale, il faut déterminer si la famille est une sphère sociale unique qui permet la circulation, dans le plus large système social, d'un médium symbolique particulier et généralisé de thèmes interchangeables - qu'on pense à la vérité, à la réciprocité ou à la solidaritée ${ }^{39}$.

On ne peut éviter ce désordre sociologique sur le sujet de la famille qu'en saisissant la réalité autonome de cette relation dans ses propres termes. Mais en quoi consiste-t-elle, cette réalité relationnelle de la famille? Est-ce la famille qui fait une promenade? Probablement pas. Imaginez, même si l'exemple est banal, un homme, une femme et un enfant qui marchent dans le parc. Si nous savons déjà que c'est la famille Tremblay, nous pouvons affirmer que nous voyons la famille Tremblay marcher. Si nous ne connaissons pas ces personnes, nous n'avons accès qu'à un nombre limité de possibilités de croisements de relations sociales, biologiques et psychiques entre eux : la question est de savoir s'il s'agit d'une famille est suspendue. Quelle est donc cette famille? Supposons qu'une fratrie, une grand-mère ou une autre personne résidant sous le même toit ne sont pas là au moment de l'obser-

39 Luhmann est ambivalent ici. Dans ses premiers écrits, influencés par Parsons, il prétend que l'amour est le médium symbolique généralisé d'échanges appartenant à la famille, mais cette opinion semble disparaître au fil du temps. L'amour comme passion n'est certainement pas un médium qui circule dans le système social en tant que moyen reconnaissable et praticable utilisé par d'autres sous-systèmes. En réalité, dans le cadre du tournant communicatif, Luhmann donne une définition tout autre des médias symboliques de celle que l'on trouve dans la théorie parsonienne. Déterminer si un tel médium peut être réciproque (et, le cas échéant, comment c'est possible) demeure une question ouverte. 
vation. Que dirions-nous? En réalité, nous voyons des individus, mais nous pensons par le biais des relations. Pour savoir si c'est véritablement une famille, nous devons constater les relations qui existent entre ces personnes. C'est en présumant une certaine relation entre eux que nous pouvons affirmer que c'est bien la famille Tremblay qui se promène. Nous supposons donc qu'il existe une certaine relation qui lie les éléments observés. Nous voyons des individus, mais nous nous exprimons en supposant des relations. Le terme "famille» indique qu'il y a relations. Que certains membres de la famille soient là ou non, le langage utilisé pour décrire l'observation au-delà des individus est essentiellement celui des relations. Les termes ont du sens seulement s'ils renvoient aux relations.

Mais tout cela ne nous indique pas la nature de cette relation qu'est la famille, celle que nous attribuons aux individus devant nous. Dans un premier temps, on peut dire de cette relation que ses termes, que nous percevons comme étant liés symboliquement, sont, en fait, quelque chose qui remplace autre chose. Ce quelque chose n'est pas fixé dans le temps, mais demeure nécessaire pour qu'il y ait relation (de parenté ou autre; peu importe, nous nous posons toujours la question). Une question s'impose : ce "quelque chose» ne serait-il rien d'autre qu'une interprétation subjective ou même une réalité objective rendue possible par un accord intersubjectif?

Bien entendu, alors que j'observe la famille Tremblay, je l'interprète avec des symboles - des signes qui représentent autre chose - en fonction d'un sens. Cet acte interprétatif est assez complexe puisqu'il comporte des perceptions, des images et des évaluations spécifiques qui sont loin d'être simples. La question suivante importe : est-ce le moi (moi-même) qui attribue une définition à la relation de la famille Tremblay, ou est-ce plutôt le nous - moi l'observateur dans une relation interpersonnelle avec d'autres, y compris les sujets observés - qui définit ce groupe comme une famille? 
Il me semble que la réponse n'est ni l'un ni l'autre. Le sens n'est jamais une conclusion entièrement subjective ou intersubjective. Il résulte plutôt du travail de toute une culture. La famille est un tissu de relations complexes (plusieurs liens condensés) qui renvoient à d'autres symboles et à des expériences vécues qui ne sont pas uniquement subjectives ni intersubjectives. En disant "je vois la famille Tremblay marcher», je me réfere à quelque chose au-delà de moi et au-delà des sujets devant moi. Il y a référence à la structure sociale qui actualise le tissu de relations complexes entre la culture, la personnalité, les normes sociales, voire les prémisses biologiques. Un tel tissu entrecroisé change certainement à travers l'histoire, mais n'est pas uniquement subjectif ou intersubjectif.

Par conséquent, la relation qu'est la famille n'est pas simplement le produit de perceptions, de sentiments et d'états mentaux intersubjectifs qui sont plus ou moins empathiques avec d'autres; elle est à la fois un fait symbolique (elle renvoie à) et structurel (elle lie) qui génère un émergent : la famille comme un Nousrelation ou un sujet relationnel ${ }^{40}$. En tant que telle, elle ne peut pas être réduite aux seuls sujets individuels qui la constituent, même si son existence dépend des sujets. C'est en eux que la relation prend vie de façon particulière. Mais l'individualisation des processus physiques et mentaux de la perception, de la sensation et de l'imagination, même quand la créativité est exprimée, ne peut pas avoir lieu sauf par le moyen d'un partage. C'est ce que nous entendons lorsque nous postulons que toute relation sociale implique un modèle culturel. Après tout, ce type de modèle s'avère une référence symbolique qui nourrit les sentiments, les émotions et les états d'âme, ceux-là même qui encouragent les individus soit à entrer et rester en relation, soit à s'en sortir et à éviter toute relation. Dans son fonctionnement, la psyché des agents individuels dépend du contexte des relations humaines et des motivations de divers ordres (rationnelles et irrationnelles, libres et forcées, intentionnelles et non intentionnelles) qui

40 Pierpaolo Donati et Margaret S. Archer, The Relational Subject, Cambridge, Cambridge University Press, 2015. 
interagissent de différentes façons. Dans le contexte de la famille en particulier, il arrive que la raison et les émotions agissent simultanément; à ce sujet, Laflamme propose la catégorie d'émoraison ${ }^{41}$. Cette interaction pour l'effet de modifier la façon dont les gens exercent leur réflexivité personnelle et relationnelle du Nous-relation. Et les diverses formes de transitions dépendent des différentes manières dont chacun peut exercer sa réflexivité par rapport, précisément, au Nous-relation ${ }^{42}$.

L'entrecroisement des relations qui caractérise la relation est ouvert à l'infini; cependant, cet état de choses risque de mener constamment vers l'indétermination. La sociologie postmoderne se définit moins par le fait d'avoir découvert cette réalité que par le fait d'avoir entrepris de comprendre et de construire le social (notamment la famille et ses réseaux sociaux internes) en tenant compte de ladite indétermination.

Ainsi, cette famille, à titre de relation sociale, est-elle indéterminée, ou alors peut-elle se référer, de manière purement contingente, à d'autres relations différentiées ad infinitum selon un cycle de déterminants qui sont ni plus ni moins des interactions réciproques? Toute expérience neutralise cette conclusion. D'un point de vue réflexif, si la relation est un tissu complexe, il doit y avoir des mécanismes de détermination. Les opérations de ces mécanismes ne sont pas strictement interactives et circulaires ad infinitum.

Encore faut-il situer ces mécanismes et déterminer leur fonctionnement. Voilà le talon d'Achille des paradigmes modernes et postmodernes. Ici, toute fracture dans la circularité venant de l'extérieur de l'interaction semble dogmatique - à l'instar de la "Vieille Europe» de Luhmann - pour les modernistes et postmodernistes. Résultat? Ils en reviennent aux notions d'Eigenvalues (valeurs autogénérées) pour soutenir que l'interruption de la

\footnotetext{
41 Simon Laflamme, Comunication et émotion. Essai de microsologique relationnelle, Paris, L'Harmattan, coll. "Logiques sociales », 1995.

42 Pierpaolo Donati, "How to Cope with Family Transitions When Society Becomes an Unbound Morphogenesis ", dans Eugenia Scabini et Giovanna Rossi (dir.), Family Transitions and Families in Transition, Milan, Vita e Pensiero, 2012, p. 29-47.
} 
circularité se fait dans un processus interactif qui établit une valeur autogénérée qui, elle, joue le rôle d'une norme régulatrice provisoire dans l'interaction en inspirant confiance plutôt qu'en imposant des contraintes.

Selon les tenants du néo-fonctionnalisme, il n'est pas possible de conceptualiser la famille comme une relation-interaction structurée par le biais du très important processus d'individualisation qui s'opère au sein des familles et entre elles, celui qui est possible grâce au mécanisme de réinsertion permettant aux acteurs d'échapper aux contraintes sans les réprimer ni les éliminer. Conséquemment, ce même processus ne permet pas d'envisager la totalité des familles comme un sous-système sociétal de la même manière qu'on dirait que la totalité des corporations est un sous-système économique ${ }^{43}$.

L'individualisation de la famille, selon Luhmann, permet de justifier l'assertion selon laquelle la totalité des familles n'a aucune fonction sociale, que les familles ne partagent désormais ni un système culturel commun, ni un médium de communication spécifique (l'amour, la solidarité), et qu'elles n’ont pas la capacité de les produire. Cette conclusion s'oppose à la réalité, comme l'ont démontré les recherches sociologiques sur les sociétés moins différenciées, mais aussi sur les sociétés complexes.

\section{La nouvelle approche relationnelle réaliste critique}

Compte tenu de l'ontologie réaliste vue plus haut, la solution aux problèmes déjà mentionnés (la difficulté de se situer par rapport à la réalité autonome des relations sociales et de définir l'objet dans des termes relationnels) implique un changement épistémologique, sans compter un paradigme et une méthodologie qui reflètent cette réorientation, qui permet l'adoption d'une pratique sui generis dans le service social.

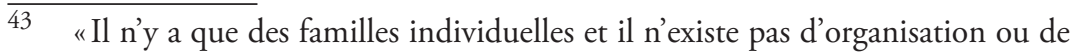
médium (l'amour) qui unifierait plusieurs familles. Et contraitement aux sociétés segmentées, il n'y a pas d'institutions qui permettraient à plusieurs familles d'opérer en tant qu'unité, du moins sous des conditions prédéterminées» (Niklas Luhmann, "Sozialsystem Familie », op. cit., p. 89). 


\section{I. Épistémologie relationnelle}

On peut résumer symboliquement le postulat fondamental de la pensée sociologique comme suit : «au commencement était la relation", phrase qu'il faut comprendre dans un sens réaliste et non relativiste. Être l'objet de connaissances humaines suppose une appartenance au réel. Il n'y a aucune séparation absolue entre la réalité objective et l'intellect humain. Avant même de s'éveiller au monde, il existe déjà une relation antérieure et précédente.

Le processus social, avec tout ce qu'il a de distinct, se déroule grâce aux relations, à travers elles et à partir d'elles. Le postulat est valable tant pour la réalité sociale (phénoménologie) que pour la théorie (observation et réflexion). Être relationnel est une caractéristique fondamentale non seulement de la réalité sociale, mais aussi de la pensée sociale. En effet, la pensée sociale a comme point de départ des aspects théoriques plutôt autonomes; viennent ensuite des aspects intermédiaires et méthodologiques et, enfin, des faits empiriques; le tout s'avère un processus réflexif continuel entre les différents mouvements et stades. Le fait de présumer que la relation existe, qu'elle est primaire et générale, et le fait de l'incorporer dans la sphère des connaissances métaphysiques, ne suppose aucunement une contingence absolue du monde social, ni non plus une ontologie niant le sujet. Au contraire, on suppose que la relation a une racine (ou référent) non contingente qui prend forme dans une situation contingente. Bien sûr, une telle racine se place en dehors de l'espace-temps de toute société, au-delà de la phénoménologie sociale concrète. Les notions telles que le bien commun, le bien public, la dignité humaine renvoient à des réalités latentes, irréductibles à leurs définitions immanentes en termes légaux ou autres. Ces réalités appartiennent aux relations entre le domaine de l'immanence et la méta-réalité ${ }^{44}$, à savoir la latence de la société. Pour voir cette réalité, il faut recourir à une matrice symbolique (finalement " théologique " parce qu'elle se réfère à la réalité ultime de la

$\overline{44}$ Roy Bhaskar, Reflections on MetaReality: Transcendence, Emancipation and Everyday Life, London, Routledge, 2012. 
même relation ${ }^{45}$ ), capable de relier les différents ordres (stratifiés) de la réalité, c'est-à-dire la réalité naturelle, pratique, sociale et surnaturelle.

D'un point de vue sociologique, seule la relation à proprement parler est nécessaire, alors que son actualisation reflète la contingence en vigueur dans la société - comment elle est, comment elle pourrait être. C'est possible, mais ce n'est pas le cas. Si les choses sont ainsi, c'est que la relation, bien qu'elle soit elle-même nécessaire, requiert aussi des déterminants historiquement précis qui sont eux-mêmes, toutefois, étant situés en dehors du système de référence, contingents ${ }^{46}$.

En ce sens, nous pouvons déclarer, par exemple, que les formes primaires de la vie sociale, dans la mesure où elles sont des relations sociales, excèdent la société; elles l'outrepassent ou la dominent de par le fait qu'elles ne sont plus une simple contingence (sur le plan notamment de la communication). Prenons la relationnalité dans le cadre de la famille, un groupe social primaire qui excède la société. Cette qualité de la relationnalité, le fait qu'elle excède la société, ne signifie pas, comme le maintient Luhmann, que la famille a, sur le plan empirique, la plus grande densité de communication détectée parmi toutes les formes d'interaction. Il existe certainement d'autres formes sociales qui témoignent d'une densité semblable. Du point de vue de la sociologie relationnelle, la famille excède la société parce qu'elle représente la nécessité d'une relationnalité complète qui exige des modèles d'organisation contingents de plus en plus complexes dans la vie quotidienne ${ }^{47}$.

45 Pierpaolo Donati, La matrice teologica della società, Soveria Mannelli, Rubbettino, 2010.

46 Emmanuele Morandi, "Introductory Outlines to Pierpaolo Donati's Relational sociology, Part 2 ", Journal of Critical Realism, vol. 10, n 1, 2011, p. 100-121.

47 L'affirmation suivante de Ted Huston et Elliot Robins nous aide à comprendre le concept d'une relationnalité complète : «Les raisons qui expliquent le fonctionnement des relations ne peuvent être comprises sans tenir compte de leur contexte écologique, un contexte qui contient des éléments historiques, économiques, culturels et physiques. Les caractéristiques psychologiques et biologiques des participants ne peuvent être ignorées" ("Conceptual and Methodological Issues in Studying Close Relationships ", Journal of Marriage 


\section{Paradigme de réseaux}

Ici, la société est comprise selon un paradigme qui n'est ni celui de la partie et du tout, ni celui de système et d'environnement, ni encore celui de l'autopoïèse, mais bien celui des réseaux. La société est envisagée comme un réseau de relations, non seulement de relations entre des nœuds, mais aussi de relations entre relations. C'est pourquoi, si nous voulons expliquer les dynamiques d'une famille de trois personnes, il ne suffit pas d'examiner les relations entre les trois nœuds; il faut aussi considérer un réseau de neuf relations aux premier, deuxième et troisième degrés. Plus nous augmentons le nombre de nœuds, plus le nombre de relations augmente jusqu'au troisième degré selon la formule ci-dessous, où $F_{k}$ équivaut au nombre de relations du degré $k ; n=$ nombre initial de nœuds $\geq 2 ; \mathrm{n}_{\mathrm{k}}=\mathrm{F}_{\mathrm{k}}-1\left(\mathrm{n}_{\mathrm{k}-1}\right) ; \mathrm{F}_{1}$ $(\mathrm{n} 1)=\mathrm{n}(\mathrm{n}-1) / 2$.

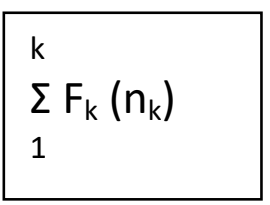

Il est important de comprendre la relation entre réseau et système. Le premier est plus général que le second, non l'inverse. Les systèmes sont une sorte de condensation et d'auto-organisation stable de réseaux, comme une vapeur ou un gaz qui se convertit en liquide et se solidifie. Avant de devenir des systèmes, les réseaux sociaux conduisent à (ou permettent le passage vers) une réalité et à des possibilités plus riches en matière de caracté-

and the Family, vol. 44, $\mathrm{n}^{\circ} 4,1982$, p. 923). Il est clair que la recherche théorique et empirique doit être sélective pour effectuer des analyses spécifiques. Il faut toutefois être conscient du regard réducteur que l'observateur scientifique peut poser sur la réalité. Et n'oublions pas que plus la prémisse est abstraite, plus elle sera susceptible de s'autovalider. De mon point de vue, la relationnalité complète est similaire à la description dense (thick description) de Clifford Geertz ("Thick Description: Toward an Interpretive Theory of Culture ", dans Clifford Geertz, The Interpretation of Cultures: Selected Essays, New York, Basic Books, 1973, p. 3-30); dans les deux cas, il y a pluralité des niveaux de discours, pluridimensionnalité et significations inépuisables. 
ristiques systémiques. C'est ici qu'il faut repenser, en des termes relationnels, la fracture actuelle entre l'analyse structuraliste et l'analyse culturelle (communicationnelle) des réseaux. L'une et l'autre voient d'un œil différent la différenciation sociale : elle est fonctionnelle pour les structuralistes, mais relationnelle du point de vue de la sociologie relationnelle. La sociologie de Simmel a, la première, rendu compte de cette différence; qu'on pense à ses études sur des cercles sociaux croisés (analyse structurale), mise en rapport avec ses Fragments of Everyday Life, où il décrit la réalité sociale comme étant composée de relations polymorphiques et magmatiques. Aujourd'hui, alors qu'on numérise la vie quotidienne, le phénomène des relations sociales a acquis des caractéristiques qui requièrent, si nous voulons comprendre la logique complexe des nouvelles formes de réseautage, un paradigme relationnel plus sophistiqué que par le passé.

\section{Pratique relationnelle}

Les implications pratiques de la sociologie relationnelle peuvent être classées selon les différentes approches que l'on adopte par rapport aux problèmes sociaux, c'est-à-dire les interventions de réseaux.

Les lignes directrices sur ces pratiques, qui servent d'appui aux politiques et services sociaux, se résument de la façon suivante :

(a) Les sujets et les objets isolés n'existent pas; il n'existe que des relations complexes entrecroisées où ils sont définis relationnellement ces sujets et objets dans leur capacité de reproduire ou de changer leur identité, ainsi que d'agir dans cette relation. Dire qu'il y a morphose et morphogénèse ne signifie pas qu'il y a relativisme et que tout peut être lu et modifié à volonté. Le problème du relativisme n'est résolu que lorsque nous définissons les relations entre différents systèmes de référence.

(b) Quand une personne intervient par rapport au sujet ou à l'objet impliqué dans un problème social, elle doit intervenir sur les relations entrecroisées dans lesquelles est incrusté 
ce sujet ou objet. L'intervention devrait viser une meilleure réflexivité personnelle et collective des acteurs en jeu dans la scène observée, et ce, en notant les effets sur le réseau que cette intervention peut provoquer.

(c) Savoir qu'une relationnalité existe entre l'observateur et l'observé, et que de cette relationnalité se dégage de l'herméneutique circulaire, ne rend pas l'intervention moins directionnelle. En effet, le cercle herméneutique peut être interrompu, de manière temporaire, par les dynamiques du réseau ${ }^{48}$.

Évidemment, ces lignes peuvent être conçues, comprises et mises en œuvre. Toutefois, il est important de ne pas donner légitimité à un réductionnisme a priori sélectif.

\section{Relationnel et systémique : synonymes?}

En retraçant l'histoire des paradigmes avec lesquels la sociologie peut comprendre la société, Luhmann discute de trois grands paradigmes systémiques :

I. Le paradigme de la partie et du tout, fondé sur l'analogie organique de la relation entre le corps et ses organes (Herbert Spencer).

II. Le paradigme du système et de l'environnement, développé par les premiers théoriciens qui ont envisagé le système social comme une relation entre les rôles institutionnalisés et tout ce qui n'est pas institutionnalisé (Talcott Parsons, par exemple).

III. Le paradigme de l'autopoïèse, qui veut que les systèmes soient fondés uniquement sur leurs propres structures et opérations (Humberto R. Maturana et Francisco J. Varela) ${ }^{49}$.

48 Pour plus de détails, voir les systèmes d'intervention sociale ODG (relational Observation-Diagnosis-Guidance) dans Pierpaolo Donati, Teoria relazione della società, op. cit., p. 346-356. 
Il ne sera pas question ici de l'évolution des théories du système, et notamment de la possibilité qu'un paradigme en remplace un autre. Je préciserai simplement que le paradigme autopoïétique n'est pas moins problématique que ses prédécesseurs, même s'il peut paraitre radical. S'il est vrai qu'un paradigme systémique ne peut plus en être un où sont liés de manière organique la partie et le tout, ce même paradigme doit toutefois, de façon adéquate, répondre autrement au problème des relations entre la partie et son tout - à condition que la perspective ne soit plus organique. Il reste à voir si les deux autres paradigmes pourront être à la hauteur; si oui, comment et à quel degré?

Voici mon hypothèse : ces deux derniers paradigmes ne répondent pas aux critères de la pensée relationnelle. D'abord, le paradigme du système et de l'environnement est une théorie de différenciation locale. Tout système se base sur la différence entre le système et l'environnement en misant sur des points limites sensibles à cette distinction; mais cela ne nous aide pas à éclairer les relations entre le système et l'environnement. On voit le même problème du côté du paradigme de l'autopoïèse, car on y étudie uniquement les rouages internes du système. Par conséquent, voici notre prochaine question : existe-t-il un paradigme qui permettrait d'élucider les relations entre le système et son environnement sans adhérer ni à la logique organique ni à la logique autoréférentielle? Je propose d'explorer cette possibilité à partir du concept de paradigme relationnel, lequel envisage les frontières entre le système et l'environnement comme un réseau de relations.

Commençons par une première question : les réseaux sociaux, ceux qui permettent une compréhension de la société contemporaine, sont-ils des systèmes sociaux?

Ceux qui répondraient oui adoptent une perspective structuraliste ou néo-fonctionnaliste ${ }^{50}$. Dans le camp adverse, on cherche une théorie des systèmes ouverts qui ont recours à un

50 Peter Blau, "Structural Sociology and Network Analysis ", dans Peter V. Marsden et Nan Lin (dir.), Social Structure and Network Analysis, Beverley Hills, Sage, 1982. 
type de cadre phénoménologique où intersubjectivité et empathie sont privilégiées (comme chez Achille Ardigò ${ }^{51}$ ). Soyons clairs : la métaphore des systèmes ouverts est utile pour souligner les limites et le réductionnisme de la métaphore des systèmes fermés du type autoréférentiel et reproductif. Cependant, la solution proposée est inutile si nous oublions que la société est comme une toile.

Il est réducteur de penser que la relation sociale entre les acteurs A et B (qu'ils soient des individus ou des groupes) est un système (ou qu'elle fait partie d'un système, d'un environnement), si par «système social» nous entendons un complexe de positions, de rôles que jouent les acteurs, où l'interaction se manifeste dans le rapport entre le comportement des acteurs et un cadre de normes régulatrices, ou d'autres contraintes, qui limitent le nombre d'actions permises pour chaque sujet à l'égard des autres. Le concept de réseau social va plus loin que la définition de système social. Comme Edward Laumann, Peter Marsden et David Prensky le maintiennent, les réseaux sociaux n'ont pas besoin de correspondre naturellement aux systèmes sociaux ${ }^{52}$. Il est clair, en effet, si l'on voit le système social comme une pluralité d'acteurs qui interagissent selon un système symbolique commun, qu'il n'y a aucune correspondance. Cependant, même en adoptant une définition plus structuraliste, il est évident que les contraintes (règles, normes) et leurs interdépendances sont parmi les caractéristiques inhérentes à la production et à la reproduction de relations préférentielles typiques des réseaux sociaux.

En d'autres mots, le concept sociologique du réseau inclut celui de système sans être réduit à celui-ci. D'une perspective réseautique, le système social est (a) un aspect analytique du réseau qui (b) manifeste ses interdépendances fonctionnelles et qui (c), là où il y a des nœuds de connexion et de disjonction,

\footnotetext{
51 Achille Ardigò, Per una sociologia oltre il post-moderno, Roma-Bari, Laterza, 1988.

52 Edward Laumann, Peter Marsden et David Prensky, "The Boundary Specification Problem in Network Analysis ", dans Ronald S. Burt et Michael J. Minor (dir.), Applied Network Analysis: A Methodological Introduction, Beverley Hills, Sage, 1983, p. 33.
} 
stabilise rétrospectivement les mécanismes et les circuits par lesquels se manifeste la phénoménologie du social. Toutefois, le réseau est aussi conducteur et locus; il est le moyen par lequel d'autres aspects, d'autres dimensions du social sont exprimés. On peut en conclure que la société est un mélange formel et informel qui exige un nouveau paradigme observationnel.

Le fait que le concept de système social ne saisisse qu'une partie des soi-disant aspects fonctionnels de la société peut décevoir. On peut donc chercher à généraliser le concept du système et à distinguer ses éléments les uns des autres pour mieux comprendre les aspects non fonctionnels et informels de l'interdépendance communicative, le communal, le mode de vie, etc. C'est ainsi qu'un système ouvert se théorise, caractérisé par le développement autosélectionné, autodirectionnel et autorégulé (plutôt que mécanique, organique ou statique) de ses parties, et opérant selon un code symbolique d'ordre supérieur et de type cybernétique ${ }^{53}$.

Une telle solution n'est pas parfaite, car les aspects informels déjà mentionnés sont nécessairement subsumés sous le système. Peu importe que ce système puisse paraître flexible grâce à ses limites contingentes qui s'occupent des ensembles flous et même des glissements; le code systémique n'en est pas moins dépendant d'une lecture mécanique - cybernétique - du social. Il s'en suit que les attributs non systémiques des relations sociales ne sont pas traités pour ce qu'ils sont. Ils sont plutôt renvoyés dans le code systémique, ou alors transposés, pour mieux les distinguer, à une sphère forcément marginale - l'irrationnel, le magique, le mythique ou une métaphysique abstraite.

En revanche, il est évident que les réseaux sociaux ne sont pas le produit d'une pure spontanéité ou d'une contingence interpersonnelle. On les compare aux sentiers sur lesquels l'individu vagabonde librement sans toutefois être maitre de ses choix.

Une étude sur les réseaux sociaux primaires ou informels de la vie quotidienne offre un point de vue éclairé pour l'observation

53 Magoroh Maruyama, "The Second Cybernetics: Deviation-Amplifying Mutual Causal Processes ", American Scientist, no 51, 1963, p. 164-179, et Walter Buckley, Sociology and Modern Systems Theory, Englewood Cliffs (NJ), Prentice-Hall, 1967. 
des relations sociales, car il ne s'agit ni d'un système, ni d'un monde vécu, mais bien de la constante interprétation de l'un dans l'autre. Par l'intermédiaire de cet itinéraire conceptuel qui enveloppe la totalité de la réflexion sociologique contemporaine, il est possible d'imaginer un quatrième paradigme qui sera en mesure de :

(a) Reconnaître que la cohérence systémique normative des deux premiers paradigmes de systèmes (celui de Durkheim et celui de Parsons) ne peut pas expliquer l'arrivée d'une société morphogénique ${ }^{54}$. La société contemporaine est caractérisée intrinsèquement par le relâchement et la fragmentation des relations sociales et par la fin d'une socialisation qui s'effectue par l'entremise des normes d'internalisation.

(b) Rejeter l'autopoï̀se comme modèle fermé et accepter la nécessité d'inclure l'autoréférentialité dans l'observation de la phénoménologie sociale, en combinaison toutefois avec l'hétéroréférentialité.

(c) Reconnaître que les acteurs sociaux ne peuvent pas être aléatoires dans leurs actions, mais que leur comportement est conditionné culturellement.

(d) Interpréter le nouvel ordre normatif de la société morphogénique, c'est-à-dire l'émergence des réseaux sociaux soumis à une logique situationnelle de circonstances (une logique relationnelle de réseaux) qui est à la fois stratégique (cognitive et guidée par des instruments), communicationnelle (expressive et dialogique) et normative (selon des valeurs généralisées).

Dans ces conditions, le concept de réseau peut constituer une forme de code métasymbolique pour le concept de système. Ce dernier doit se généraliser davantage et démontrer une différen-

54 Margaret S. Archer (dir.), Late Modernity. Trajectories towards Morphogenic Society, Dordrecht, Springer, 2014. 
ciation réflexive. Ce n'est qu'ainsi que nous pouvons analyser les réseaux sociaux et en comprendre pleinement la valeur à la fois formelle et informelle.

\section{Vaincre le fonctionnalisme grâce à la sociologie relationnelle}

Pour comprendre réellement la pensée relationnelle que je propose, il faut la voir comme une rupture critique par rapport à la pensée fonctionnaliste, surtout les versions de Durkheim et de Luhmann, en passant par Parsons.

$\mathrm{Au}$ cours du vingtième siècle, l'analyse fonctionnaliste est demeurée en arrière-plan, véritable leitmotiv, et s'est avérée l'infrastructure paradigmatique derrière la théorie et la recherche empirique en sociologie et dans d'autres sciences sociales. Pour Kingsley Davis, l'analyse fonctionnaliste n'est ni plus ni moins ce qui se fait dans toute science. Il est erroné de penser, selon lui, qu'il y a autre chose que l'analyse fonctionnaliste ${ }^{55}$. En réalité, c'est le contraire qui est vrai : le réductionnisme que favorise le fonctionnalisme est de plus en plus évident. Examinons les phases principales selon lesquels il s'est développé.

(I) Dans la première étape du fonctionnalisme, Durkheim réduit les relations sociales à desfonctions. Les entités sociales ne sont pas définies selon leur réalité indéniable, mais plutôt selon les fonctions qu'elles assurent dans la société. Ces fonctions, vues comme des rôles qui correspondent à la division du travail, vont devenir synonymes des relations sociales. Dès le début, cette conception de la relationnalité est caractérisée de manière positiviste. Dans ses célèbres Règles de la méthode sociologique, Durkheim a conçu certaines hypothèses de base.

55 Kingsley Davis, "The Myth of Functional Analysis as a Special Method in Sociology and Anthropology ", American Sociological Review, vol. 24, n 6, 1959, p. 758. 
(a) Il remplace la notion de la fonction avec celle du but. Le finalisme devait être banni de la sociologie. Lorsqu'il explique les règles des faits sociaux, Durkheim affirme que

«Nous nous servons du mot de fonction de préférence à celui de fin ou de but, précisément parce que les phénomènes sociaux n`existent généralement pas en vue des résultats utiles qu'ils produisent. Ce qu'il faut déterminer, c'est s'il y a correspondance entre le fait considéré et les besoins généraux de lıorganisme social et en quoi consiste cette correspondance, sans se préoccuper de savoir si elle a été intentionnelle ou non. Toutes ces questions d'intention sont, drailleurs, trop subjectives pour pouvoir être traitées scientifiquement ${ }^{56}$.

L'analogie organique doit être garante de l'objectivité positive de l'analyse sociologique ${ }^{57}$.

(b) Sur cette base, l'analyse sociologique devrait expliquer le phénomène selon deux procédures. Premièrement, l'analyse devrait souligner le lien entre les parties de la société et sa totalité; deuxièmement, elle devrait mettre en évidence les liens entre ces différentes parties. Ces deux opérations doivent être effectuées en tenant compte des fonctions spécialisées dont s'acquittent les parties au service du tout.

Selon Kingsley Davis, le non-fonctionnalisme implique toujours soit une sorte de réductionnisme, soit un empirisme plutôt approximatif. Le premier peut prendre la forme d'un psychologisme (le statut de la conscience individuelle est expliqué par le social), d'un biologisme (réduction du social à des facteurs

\footnotetext{
56 Émile Durkheim, Les règles de la méthode sociologique, Paris, Presses universitaires de France, 16 édition, 1967 [1894], Chicoutimi, Les classiques des sciences sociales, coll. "Les auteur(e)s classiques ", http://classiques.uqac.ca/ classiques/Durkheim_emile/regles_methode/durkheim_regles_methode.pdf, p. 58.

57 Émile Durkheim, De la division du travail social (8 édition, Paris, Presses universitaires de France, coll. "Bibliothèque de philosophie contemporaine ", 1967 [1893], Chicoutimi, Les classiques des sciences sociales, coll. " Les auteur(e)s classiques ", http://classiques.uqac.ca/classiques/Durkheim_emile/ division_du_travail/division_travail.html). Robert K. Merton aussi affirmera plus tard que les fonctions sociales se réfèrent aux conséquences objectives observables, non aux dispositions subjectives (objectifs, motifs, fonctions) (Social Theory and Social Structure, New York, Free Press, 1968, p. 78).
} 
génétiques), ou d'un déterminisme (économique, technologique, etc.) qui fonctionne de la même façon. Le second comprend uniquement une manipulation non théorique de données, au contraire de l'analyse fonctionnelle systémique, qui utilise un modèle interprétatif, et non seulement des relations statistiques ou des données historiques. Comme nous le rappelle Davis, la physiologie reste le modèle de référence pour la quasi-totalité des auteurs fonctionnalistes, qu'on pense à Alfred Radcliffe-Brown, Bronislaw Malinowski, Raymond Firth et Robert K. Merton.

Apparemment, il est facile pour le langage scientifique de revenir au fonctionnalisme du sens commun : "parler de la fonction d'une institution vis-à-vis de la société (ou vis-à-vis d'une autre institution) est une façon de déterminer le rôle de l'institution dans le système duquel elle relève ${ }^{58}$. " Cette première version du fonctionnalisme se veut une description et une explication de phénomènes selon la perspective d'un système de raisonnement qui, en toute vraisemblance, ne serait aucunement lié à un système de nature homologue. Ce qui est particulier dans le cas de la sociologie, c'est le sujet, c'est-à-dire (selon Davis) la société.

Que sont devenues la compréhension et l'interprétation? L'analyse fonctionnaliste montre déjà de grandes difficultés. Non seulement le sujet humain est-il expulsé (avec ses motifs internalisés et ses expériences), mais on entrevoit maintenant la culture comme si elle était naturaliste. Inévitablement, la société devient mécanique. Si les tendances susmentionnées se maintenaient, la relation sociale serait réduite à une simple structure au sein de laquelle la culture ne serait qu'un ensemble limité de possibilités. Voilà les problèmes et les ambiguïtés que la première étape du fonctionnalisme n'a pu résoudre. Même si la société se présente comme un organisme culturel, elle est étudiée comme un organisme naturel en évolution. On n’a jamais pu renoncer à cette ambiguïté générique.

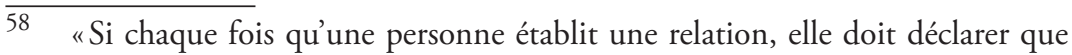
ceci a telle ou telle fonction, cela devient ennuyeux. Pourquoi ne pas simplement dire que le cœur pompe le sang dans tout le système? " (Kingsley Davis, op. cit., p. 772). 
(II) La deuxième étape du fonctionnalisme se résume à la théorie de Talcott Parsons, qui présente un fonctionnalisme fidèle aux principes structuraux de Durkheim. Toutefois, s'inspirant de Weber, il a essayé d'incorporer l'accent sur l'intentionnalité et de reprendre les facteurs non rationnels de Vilfredo Pareto (cette soi-disant convergence entre Durkheim, Weber et Pareto sur la théorie de l'action). Conséquemment, la théorie sociale devrait renoncer à toute tentative d'établir ou de faire avancer des lois scientifiques exactes.

Le fonctionnalisme de Parsons, du moins dans sa phase initiale, se caractérise par le désir d'accorder au sujet humain son rôle de sujet d'action et de confier à la culture une grande liberté et une latence (valeurs ultimes). On croirait donc qu'il est possible d'affirmer que la relation peut être redéfinie d'une manière non réductionniste. Cependant, Parsons n'a jamais été aussi loin. En effet, il a été de plus en plus difficile, sinon impossible, pour le fonctionnalisme traditionnel de conserver le sujet et pour la culture de les élever dans le cadre de la théorie sociologique.

Après l'abandon de l'approche systémique et sa renaissance biologique et (surtout) cybernétique, le fonctionnalisme a emprunté la voie d'une relationnalité de plus en plus structuraliste et relationnelle. Le système prend la place du sujet et la culture est réduite à un sous-système.

Comparé au fonctionnalisme primitif et naïf du XIX siècle, celui de Parsons était plus relationnel encore et marquait une transition vers le paradigme du système et de l'environnement au détriment du paradigme de la partie et du tout. Avec cette transformation, la relation sociale est devenue un lien entre les rôles et les statuts, ainsi qu'un dispositif d'échange entre le système et son environnement. Au cour de la relation se trouve le système $e^{59}$.

59 Talcott Parsons, op. cit. 
Dès lors, l'intégration sociale et l'intégration systémique n'étaient plus le fondement de la théorie. Au contraire, les deux s'opposaient, étant presque incompatibles, comme si l'une était examinée depuis la perspective de l'environnement de l'autre. C'est ainsi que la relation sociale fut divisée en deux parts, une dimension intersubjective (intégration sociale) et une dimension fonctionnelle (intégration systémique), lesquelles ne peuvant que s'opposer et se limiter mutuellement. Dans une large mesure, les fonctionnalistes classiques n'ont pas pu se défaire de ces difficultés.

(III) La troisième vague du fonctionnalisme touche le travail de Luhmann, qui déploie le plus grand effort pour traiter du fonctionnalisme classique de Parsons et de ses ambivalences non résolues. Ce faisant, Luhmann tire des conclusions plutôt radicales sur ce qui caractérise le fonctionnalisme depuis ses débuts.

Le fil principal du fonctionnalisme théorique - le concept de système - est devenu l'axiome fondamental de Luhmann pour redéfinir tous les concepts de l'approche théorique. La différence entre système et environnement, introduite par Parsons, est élevée au statut de définition intégrale de la théorie, comme la distinction entre identité et différence. Ainsi, il adopte un nouveau paradigme fonctionnaliste qui était implicite, en quelque sorte, depuis le départ : le paradigme autopoḯtique autoréférentiel.

Je dois préciser qu'une certaine conception de la relation se trouve à la source de ce changement, tout comme elle fut présente dans la transition de Durkheim à Parsons. Selon Luhmann, la théorie requiert des concepts formels établis au niveau des relations concernées ${ }^{60}$. En effet, il est regrettable que Luhmann ait conçu des relations sociales formalistes radicales qu'on a conséquemment méprises pour des relations logiques. Ce qui était sous-entendu, c'est que la relation, comme dans le domaine de la logique, devait tout d'abord référer à elle-même pour être traitée; la relation est ici un concept primitif, et donc une

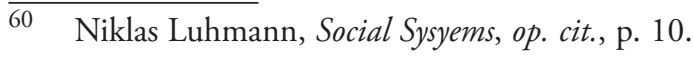


construction autoréférentielle plutôt qu'une réalité émergente. Selon la sociologie de Luhmann, cela veut dire notamment que les relations sociales ne peuvent pas être soumises à des analyses empiriques, qu'elles soient explicatives ou interprétatives, qui permettraient d'en relever les composantes et les interactions.

Le passage du paradigme du système et de l'environnement au nouveau paradigme autoréférentiel est caractérisé par une conception de la relation causa sui (la relation par manière de l'explication causale de soi) ${ }^{61}$.

Ici, la relationnalité est le sujet d'une redéfinition radicale que l'on peut décrire comme suit :

i. Les composantes biologiques, psychiques et sociales de ce monde doivent être observées et expliquées par une utilisation relationnelle logique de la relation. Nous ne pouvons rien faire sans la relation - elle est partout. Dans les systèmes basés sur le sens, la relation devient pratiquement l'équivalent du sens, ou mieux : le sens est la relation elle-même logiquement parlant.

ii. La théorie doit élaborer des concepts qui possèdent une capacité relationnelle. Par exemple, le concept de la complexité doit être perçu comme étant complexe en soi. Cette perception représente, par-dessus tout, la capacité de tenir compte d'un nombre illimité de relations (et d'éléments que supposent ces relations) ${ }^{62}$.

61 Selon Luhmann, «les constructions théoriques relativement simples étaient encore possibles dans le contexte de la théorie du système et d'environnement. Par exemple, la théorie pouvait être interprétée comme une extension simple de relations causales : il fallait considérer autant les facteurs internes qu'externes dans toute explication causale; le système et l'environnement sont alliés dans une sorte de coproduction. La théorie des systèmes autoréférentiels contourne ce modèle causal. Elle envisage la causalité (sans oublier la déduction logique et toutes les sortes d'asymétrisation) comme une forme d'organisation d'autoréférentialité, et elle explique la différence entre système et environnement en postulant que seuls les systèmes autoréférentiels peuvent se créer la possibilité d'ordonner les causalités par la distribution dans le système et l'environnement» (ibid., p. 9-10).

62 Luhmann affirme que tout état des choses complexe se fonde sur une sélection de relations entre ses éléments, qu'il utilise pour se constituer et se maintenir. 
iii. En même temps, la relation devient la réduction de la complexité. Cela veut dire qu'elle doit être conceptualisée comme un système émergent ${ }^{63}$. En d'autres mots, c'est la relation qui, logiquement parlant, réduit et amplifie la complexité.

iv. La relation sociale n'est désormais plus l'expression des sujets et de leurs actions. L'idée d'une action réciproque et mutuelle est réduite à la communication. En même temps, l'individu ne peut être sûr qu'on le comprend, car chaque communication produit des subjectivités autoréférentielles difficiles à saisir. Qu'est-ce qui fait que l'unité de la relation peut unifier une pluralité de systèmes autoréférentiels? La question est problématique. Les relations sociales sont sujettes à une temporalisation radicale et sont ainsi marquées par une circularité. Les séquences conjonctives établies deviennent de moins en moins prévisibles.

C'est ainsi que le fonctionnalisme soutient un contingentisme omniprésent, qui n'est mitigé que par la nécessité pragmatique de structurer (sur les plans séquentiel et temporaire) le système. En fin de compte, le système apparaît comme une relationnalité fonctionnelle systémique de relations fonctionnelles. Le fonctionnalisme s'avère dès lors fondé sur des sables mouvants.

Pourtant, dans les sciences sociales, le fonctionnalisme ne montre aucun signe de déclin. En dépit de sa critique de Parsons, qui aurait sous-estimé le Lebenswelt, Jürgen Habermas a finalement évalué favorablement une grande partie de la théorie parsonnienne. Et parmi les prétendues théories relationnelles, plusieurs travaillent à partir d'une conception fonctionnelle pragmatique de la relation sociale, laquelle est envisagée comme étant des transactions sociales de réseaux ${ }^{64}$.

La sélection positionne et qualifie les éléments, bien que d'autres relations sont possibles (ibid., p. 25). Selon lui, nous devrions parler d'une réduction dans la complexité si le cadre des relations qui forme un nœud complexe se reconstruit par un deuxième nœud ayant moins de relations (ibid., p. 26).

64 Ibid., p. 108.

Christopher Powell et François Dépelteau (dir.), Relational Sociology. Ontological and Theoretical Issues, New York, Palgrave Macmillan, 2013; 
Tout cela nous porte à conclure que pour les sciences sociales, à l'heure actuelle, l'analyse fonctionnelle systémique n'est pas une approche ou une méthode parmi d'autres; elle sous-tend tout discours scientifique. Comparée à la théorie d'Habermas, celle de Luhmann a eu l'avantage de ne pas s'opposer à l'analyse fonctionnelle systémique, mais plutôt de l'intégrer, d'être sur la même longueur d'onde, ce qui lui a permis d'entrevoir une nouvelle perspective du postmoderne; Habermas, lui, a voulu la récupérer, ou du moins la rendre compatible avec son propre point de vue normatif (son idéal de la modernité).

À titre de code symbolique et de manière d'envisager l'univers, l'analyse fonctionnelle systémique a érodé les traditions culturelles partout dans le monde et a démontré une capacité de se régénérer continuellement grâce à des formulations de plus en plus sophistiquées.

Certains croient que les traditions culturelles peuvent se raviver et se venger. Quel espoir ces contre-pressions ont-elles de freiner l'élan du fonctionnalisme? Presque aucun, il semblerait. Le fonctionnalisme les considère comme de simples illusions, alors que la globalisation fonctionnelle fait des avancées à peu près partout. Ainsi, il est difficile de savoir pourquoi le fonctionnalisme, en dépit des limites qu'on lui connaît, jouit d'un tel succès - du moins en apparence.

De Durkheim à Parsons et ensuite à Luhmann, le fonctionnalisme change constamment, mais démontre une résistance surprenante. Il y a plusieurs années, Alvin Goulder a déclaré qu’il fallait accepter la mort du fonctionnalisme, et il n'était pas le seul. De toute évidence, cette mort se fait attendre, même aujourd'hui. Mais pourquoi? Quelle est la force du fonctionnalisme?

Il y a d'abord, sans aucun doute, le fait que le fonctionnalisme se distancie des jugements de valeur. Théoriquement, il suppose un quantum (variable) de relativisme culturel et épistémologique qui implique un certain degré (variable) de relativisme moral; voilà sa force première. Puisqu'il n'est pas figé sur une seule 
position morale, le fonctionnalisme est compatible avec des positions des plus diverses, ce qui fait qu'il convient à plusieurs approches. De plus, il englobe tous les concepts et instruments qui ne nécessitent pas de prise de position.

Ensuite, il y a le fait que le fonctionnalisme est une méthode qui compte sur des catégories logiques au fort pouvoir d'abstraction. Le concept de fonction, analogue à celui des mathématiques, permet un nombre presque infini de jeux, d'extrapolations et d'applications.

En fin de compte, il est clair que l'analyse fonctionnelle occupe le même rôle en sociologie que les mathématiques en économie. Elle est, de toute évidence, indispensable. Cependant, les mathématiques n'ont pas l'importance qu'on leur prête; elles ne permettent même pas de comprendre et d'expliquer l'économie, se limitant à la rendre plus facile à calculer et à anticiper. On peut en dire autant de l'analyse fonctionnelle dans le contexte des sciences sociales.

Ma sociologie relationnelle réaliste est justement une tentative de combler les lacunes du fonctionnalisme. Je propose que les sciences sociales sont supra-fonctionnelles.

Aussi fascinante soit-elle, la solution fonctionnaliste n'a pas résolu le problème de sa relation au non-fonctionnel. Les difficultés liées à l'interprétation, laquelle est nécessaire pour considérer le non-fonctionnel, ne voient aucune solution dans l'analyse fonctionnaliste. C'est la déficience majeure de cette approche, car aucun fonctionnaliste n'a pu démontrer le côté humain de ce qu'elle laisse de côté.

Le problème est que le fonctionnalisme mène au non-fonctionnalisme, tout comme les mathématiques en économie nous poussent à admettre l'existence de ce qui, dans le système économique, ne peut être relevé par la quantification mathématique. Pareillement, l'analyse fonctionnaliste ne peut pas faire face à son contraire. Comment Luhmann pourrait-il expliquer l'implosion du sens, l'appel à la justice, l'utopie de plusieurs mouvements sociaux et de leurs dynamiques, le partage non réciproque, l'acte gratuit ou le refus de communiquer? Comment expliquer la 
créativité de l'être humain, les premiers pas d'une synergie combinatoire et l'irruption de nouvelles significations si l'on ne se fie à rien d'autre qu'un fonctionnalisme autoréférentiel? Doit-on accepter que ce ne sont que de nouvelles connexions activées par la variabilité causale?

Il est impossible d'inclure ou d'exclure la réalité non fonctionnelle si notre perspective est fonctionnaliste. Pour résoudre ce problème, il doit être possible d'observer le fonctionnement des principes fonctionnels de définition d'un point de vue plus large - celui de la sociologie relationnelle :

o Au-delà des dimensions matérielles, psychiques et sociales, le sens a une dimension culturelle. La dimension temporelle du sens est constituée de ces quatre autres types de sens, lesquels sont autant de dimensions dans la mesure où chacun a son contenu et une dynamique qu'on lui reconnaît (le temps change le sens).

o L'action est un acte à la fois intentionnel et émergent.

o Le système émerge d'une toile de relations par des processus de morphostate et de morphogenèse.

o La complexité n'est pas uniquement un synonyme de contingence, mais une combinaison de nécessité, de contingence, de structure et d'événement.

o Les relations sociales sont supra-fonctionnelles.

À partir de ce point de vue plus large, il est possible de garder le meilleur de l'analyse fonctionnelle et de l'intégrer à l'analyse relationnelle, qui s'avère une théorie de la société plus convenable.

La solution n'est pas de nier l'importance de l'analyse fonctionnaliste, mais de l'inclure dans un paradigme plus large où la relation devient le principe fondamental. En sociologie, l'observation détermine si l'acteur, l'événement, la structure ou l'action sociale peut interagir et comment il le fait.

Pour le premier fonctionnalisme, l'équation $y=f\left(x_{i}\right)$ est valide, où $x_{j}$ sont des facteurs variables. Pour le deuxième fonctionnalisme, $y=f\left(x_{i}, r_{j}\right)$ est valide, où la relation entre les facteurs $\left(r_{j}\right)$ est introduite comme une variable additionnelle. Pour le troisième 
fonctionnalisme, $y=f\left(x_{i}, r_{j}, r_{n} r_{j}\right)$ est valide, où la relationnalité des relations $\left(r_{n} r_{j}\right)^{65}$ est introduite comme une variable.

L'analyse relationnelle ne nie pas les processus scientifiques que ces reformulations paradigmatiques illustrent, mais au lieu de $f$, elle insérerait la relation $R$, qui équivaut à une référence complexe et à des liens complexes qui ne sont pas nécessairement fonctionnels : $y=R\left(x_{i}\right) ; y=R\left(x_{i}, r_{j}\right) ; \mathrm{y}=R\left(x_{i}, r_{j}, r_{n} r_{j}\right)$. En sociologie, l'opérateur relationnel $R$ est une relation sociale dont les fonctions et le sens général ne sont pas discernables.

Dire que l'entité, variable ou non, $y$ (comportement, structure, événement, etc.), dépend d'autres variables $\left(x_{i}\right)$, cela sous-entend qu'on analyse l'opérateur $R$ qui interagit avec eux, d'une manière complexe et typiquement supra-fonctionnelle, par l'entremise des relations entre des éléments $\left(r_{j}\right)$ et des relations entre ces relations $\left(r_{n} r_{j}\right)$. On ne peut voir de telles relations comme étant logiques que sur un plan abstrait et formalisé, car les systèmes fondés sur le sens (où le sens est conceptualisé comme une sélection significative) sont d'abord et avant tout des schémas culturels interprétés par des acteurs.

\section{La relationnalité : le jeu des jeux}

Pour plusieurs sociologues, la connaissance orientée vers l'action reste une façon d'observer la relation entre, d'un côté, les acteurs et, de l'autre, les faits, ou encore entre différentes variables comme les actions, le pouvoir, l'argent, les classes, etc., plutôt qu'une façon de voir les phénomènes sociaux comme des relations ou, mieux encore, des relations de relations. C'est ainsi que tout s'effondre, se réduit à du relativisme (relationnisme) et nuit à l'analyse des relations sociales.

Lorsque la sociologie a développé une pensée relationnelle, celle-ci a eu un caractère positiviste ou idéaliste, et rarement réaliste dans le sens du réalisme critique, analytique et relationnel. Or, ce type de réalisme permet de comprendre et d'expliquer le

65 Par relationnalité, j'entends une réalité de la relation à double visage : l'expression reflète le fait qu'il y ait une réalité dans la relation; les deux termes qui sont liés (ego et alter) existent de par leur connexion réciproque (Pierpaolo Donati, L'enigma della relazione, op. cit., p. 96). 
monde social, non pas en le matérialisant ou le subjectifiant, mais bien en lui accordant son caractère objectif et en reconnaissant son antériorité par rapport aux acteurs et aux agents, sa construction hic et nunc, et le fait qu'il acquiert une réalité déterminée qui repose sur un niveau méta par rapport aux perceptions subjectives des acteurs concernés.

Dans tous les cas, admettre, sur le plan épistémologique, que tout sujet d'étude est socialement construit ne suffit pas pour conclure qu'il est uniquement un produit social, car la réalité est stratifiée en différents niveaux et est une création, un jeu relationnel entre diverses sphères (naturelle, sociale, pratique et transcendantale). Le fait de croire que les relations sociales sont restreintes au niveau de la performance communicationnelle ou fonctionnelle donne lieu à des formes de sociologisme injustifiables. Pour arriver à vraiment observer de manière critique et réflexive ce qui caractérise la réalité tout autour, et ce que la sociologie interprète et comment elle s'interprète elle-même, nous avons besoin d'une épistémologie relationnelle adéquate autrement dit, un système d'observation non autoréférentiel, capable d'articuler autoréférence et hétéroréférence.

Dans les sciences sociales, on ne peut ni observer, ni comprendre, ni encore expliquer le sujet de l'action en soi : il faut le faire par l'entremise des relations sociales, à l'intérieur d'elles ou avec elles. Le relationnisme postmoderne peut être transcendé, mais seulement par une lecture globale et attentive des relations sociales qui est multidirectionnelle et supra-fonctionnelle relationnelle, donc ${ }^{66}$. Le sujet humain, grâce aux relations sociales, redevient un agent conséquent, la source normative de la relation - compte tenu du fait que l'action est normative. Postuler que l'action est normative veut dire qu'elle nécessite inévitablement une interprétation, qu'elle est ainsi «herméneutique». Toutefois, il ne faut pas oublier que les relations sociales excèdent la volonté et le sens des acteurs.

66 Pierpaolo Donati, "Transcending the Human: Why, Where, and How? " dans Ismael Al-Amoudi et Jamie Morgan (dir.), Ex Machina: Realist responses to posthuman society, Routledge, Londres et New York, 2018 (sous presse). 
Nous voulons comprendre les faits sociaux dans leur réalité, mais cela n'est possible que par la voie des relations :

1. La relation est la clé pour entrer dans la réalité sociale, mais également pour en sortir, la connaissance étant une relation utilisée par un observateur qui veut comprendre ce qui est observé (aperçu, implication) et ce qui en résulte (détachement).

2. La relation n'élimine pas les éléments reliés, mais fait appel à eux, les étudie et les exprime.

3. La relation n'est pas une pure abstraction - une pure forme de communication -, mais est ancrée dans le concret.

4. Il s'en suit que l'entité relationnelle émergente, qui inclut les pensées humaines, peut être dichotomique ou duale dans des circonstances extrêmes. Normalement, elle est dotée d'une structure de réseaux qui connecte, qui lie et qui crée des interdépendances, sans compter des conflits et des tensions.

5. Les normes et les règles sont une façon inévitable et nécessaire de réguler, sous des conditions normales, la contingence de situations non prédéterminées socialement.

Pour conclure, c'est bien la relation, non pas la dualité, l'ambivalence absolue ou autre chose, qui est le jeu suprême des jeux. Cela dit, elle ne se réduit pas, cependant, au jeu. Nous ne pouvons pas transposer ce que Ludwig Wittgenstein déclarait à propos du jeu linguistique dans son essai De la certitude : «[...] quelque chose d'imprévisible. J'entends par là qu'il n'est pas fondé; qu'il n'est ni rationnel, ni non rationnel. Il est là tout simplement - comme notre vie ${ }^{67}$ ». Nous avons tendance, dans la vie de tous les jours, à croire que les relations sociales suivent des règles vagues, obscures ou ambiguës; nous avons l'habitude de polariser, de penser en codes binaires (dedans/dehors, symétrique/asymétrique, etc.). Cela nous permet de réduire à l'essentiel notre réalité. Cependant, les relations sociales ne peuvent pas, à long terme, être incertaines, ambiguës, ou dichotomiques sur le plan structural. Leur tâche est d'outrepasser l'ambiguïté et la dichotomie, même si elles manifestent continuellement ces traits.

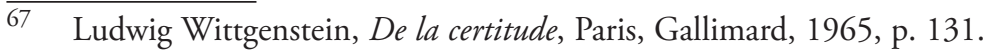




\section{Bibliographie}

Alexander, Jeffrey C., Twenty Lectures. Sociological Theory Since World War II, New York, Columbia University Press, 1987.

Archer, Margaret S. (dir.), Conversations on Reflexivity, Londres, Routledge, 2010.

Archer, Margaret S. (dir.), Late Modernity. Trajectories towards Morphogenic Society, Dordrecht, Springer, 2014.

Archer, Margaret S., The Reflexive Imperative in Late Modernity, Cambridge, Cambridge University Press, 2012.

Ardigò, Achille, Per una sociologia oltre il post-moderno, Roma-Bari, Laterza, 1988.

Bagaoui, Rachid, "Un paradigme systémique relationnel est-il possible? Proposition d'une typologie relationnelle ", Nouvelles perspectives en sciences sociales, vol. 3, $\mathrm{n}^{\circ}$ 1, 2007, p. 151-175.

Bhaskar, Roy, Reflections on MetaReality: Transcendence, Emancipation and Everyday Life, London, Routledge, 2012.

Bhaskar, Roy, The Possibility of Naturalism: A Philosophical Critique of the Contemporary Human Sciences, Brighton, The Harvester Press, 1979.

Blau, Peter, "Structural Sociology and Network Analysis ", dans Peter V. Marsden et Nan Lin (dir.), Social Structure and Network Analysis, Beverley Hills, Sage, 1982.

Buch-Hansen, Hubert, "Social Network Analysis and Critical Realism ", Journal for the Theory of Social Behaviour, vol. 44, $\mathrm{n}^{\circ}$ 3, 2013, p. 306-325.

Buckley, Walter, Sociology and Modern Systems Theory, Englewood Cliffs (NJ), Prentice-Hall, 1967.

Burt, Ronald S., Structural Holes. The Social Structure of Competition, Cambridge (MA), Harvard University Press, 1992.

Burt, Ronald S., Toward a Structural Theory of Action. Network Models of Social Structure, Perception, and Action, New York, Academic Press, 1982.

Cook, William L. et Albert Dreyer, "The Social Relations Models", Journal of Marriage and Family, vol. 46, n 3, 1984, p. 679-687.

Davis, Kingsley, "The Myth of Functional Analysis as a Special Method in Sociology and Anthropology ", American Sociological Review, vol. 24, n 6, 1959, p. 757-772.

Dépelteau, François, «Relational Sociology, Pragmatism, Transactions, and Social Field ", International Review of Sociology, vol. 25, n 1, 2015, p. $45-64$. 
Dépelteau, François et Christopher Powell (dir.), Applying Relational Sociology. Relations, Networks, and Society, New York, Palgrave Macmillan, 2013.

Donati, Pierpaolo, «L'analisi Relazionale: Regole, Quadro Metodologico, Esempi ", dans Pierpaolo Donati (dir.), Sociologia. Una Introduzione allo Studio della Società, Padova, Cedam, 2006, p. 213-227.

Donati, Pierpaolo, L'enigma della relazione, Milan-Udine, Mimesis edizioni, 2015.

Donati, Pierpaolo, «How to Cope with Family Transitions When Society Becomes an Unbound Morphogenesis ", dans Eugenia Scabini et Giovanna Rossi (dir.), Family Transitions and Families in Transition, Milan, Vita e Pensiero, 2012, p. 29-47.

Donati, Pierpaolo, Introduzione alla sociologia relazionale, $2 e$ édition augmentée, Milan, Franco Angeli, 1986 [1983].

Donati, Pierpaolo, La matrice teologica della società, Soveria Mannelli, Rubbettino, 2010.

Donati, Pierpaolo, "Morphogenesis and Social Networks: Relational Steering not Mechanical Feedback ", dans Margaret S. Archer (dir.), Social Morphogenesis. Dordrecht, Springer, 2013, p. 205-231.

Donati, Pierpaolo, Relational Sociology. A New Paradigm for the Social Sciences, Londres et New York, Routledge, 2011.

Donati, Pierpaolo, La società dell'umano, Genova-Milano, Marietti, 2009.

Donati, Pierpaolo, Teoria relazione della società, Milan, Franco Angeli, 1991.

Donati, Pierpaolo, "Transcending the Human: Why, Where, and How? " dans Ismael Al-Amoudi et Jamie Morgan (dir.), Ex Machina: Realist responses to posthuman society, Routledge, Londres et New York, 2018 (sous presse).

Donati, Pierpaolo, "What Does a "Good Life" Mean in a Morphogenic Society? The Viewpoint of Relational Sociology ", dans Margaret S. Archer (dir.), Morphogenesis and Human Flourishing, Dordrecht, Springer, 2017, p. 137-161.

Donati, Pierpaolo et Margaret S. Archer, The Relational Subject, Cambridge, Cambridge University Press, 2015.

Durkheim, Émile, Les règles de la méthode sociologique, Paris, Presses universitaires de France, 16 édition, 1967 [1894], Chicoutimi, Les classiques des sciences sociales, coll. «Les auteur(e)s classiques », http:// classiques.uqac.ca/classiques/Durkheim_emile/regles_methode/ durkheim_regles_methode.pdf. 
Durkheim, Émile, De la division du travail social, $8^{\mathrm{e}}$ édition, Paris, Presses universitaires de France, coll. "Bibliothèque de philosophie contemporaine ", 1967 [1893], Chicoutimi, Les classiques des sciences sociales, col. "Les auteur(e)s classiques ", http://classiques.uqac.ca/ classiques/Durkheim_emile/division_du_travail/division_travail.html.

Emirbayer, Mustafa, "Manifesto for a Relational Sociology ", American Journal of Sociology, vol. 103, $\mathrm{n}^{\circ}$ 2, 1997, p. 281-317.

Etxeberria, Arantza, «Autopoiesis and Natural Drift: Genetic Information, Reproduction, and Evolution Revisited ", Artificial Life, n 10, 2004, p. 347-360.

Foerster, Hans von, Observing Systems, Seaside (CA), Intersystems Publications, 1984.

Geertz, Clifford, "Thick Description: Toward an Interpretive Theory of Culture ", dans Clifford Geertz, The Interpretation of Cultures: Selected Essays, New York, Basic Books, 1973, p. 3-30.

Halas, Elżbieta, "Through Social Values to the Reinterpretation of Sociology's Ethical Neutrality ", Sociologia e Politiche Sociali, vol. 19, $\mathrm{n}^{\circ} 1,2016$, p. 67-79.

Huston, Ted et Elliot Robins, Elliot, "Conceptual and Methodological Issues in Studying Close Relationships ", Journal of Marriage and the Family, vol. 44, no 4, 1982, p. 901-925.

Laflamme, Simon, Communication et émotions. Essai de microsociologie relationnelle, Paris, L'Harmattan, coll. «Logiques sociales », 1995.

Laflamme, Simon, "Sciences sociales et approche relationnelle ", Nouvelles perspectives en sciences sociales, vol. $5, \mathrm{n}^{\circ} 1,2009, \mathrm{p} .79-85$.

Laumann, Edward, Peter Marsden et David Prensky, "The Boundary Specification Problem in Network Analysis ", dans Ronald S. Burt et Michael J. Minor (dir.), Applied Network Analysis: A Methodological Introduction, Beverley Hills, Sage, 1983, p. 18-34.

Luhmann, Niklas, Social Systems, Stanford, Stanford University Press, 1995.

Luhmann, Niklas, "Sozialsystem Familie », dans System Familie, 1, 1988, p. 75-91.

Maruyama, Magoroh, "The Second Cybernetics: Deviation-Amplifying Mutual Causal Processes ", American Scientist, n ${ }^{\circ}$ 51, 1963, p. 164-179.

Maturana, Humberto R. et Francisco J. Varela, The Tree of Knowledge. The Biological Roots of Human Understanding, édition révisée, Boston, Shambhala Publications, 1987.

Merton, Robert K., Social Theory and Social Structure, New York, Free Press, 1968. 
Morandi, Emmanuele, "Introductory Outlines to Pierpaolo Donati's Relational sociology, Part 1 ", Journal of Critical Realism, vol. 9, n² 2, 2010, p. 208-226.

Morandi Emmanuele, "Introductory Outlines to Pierpaolo Donati's Relational sociology, Part 2 ", Journal of Critical Realism, vol. 10, $\mathrm{n}^{\circ} 1$, 2011, p. 100-121.

Parsons, Talcott, The Social System, New York, Free Press, 1951.

Powell, Christopher, "Radical Relationism: A Proposal ", dans François Dépelteau et Christopher Powell (dir.), Conceptualizing relational Sociology: Ontological and Theoretical Issues, New York, Palgrave, p. 187-207.

Powell, Christopher and François Dépelteau (dir.), Relational Sociology. Ontological and Theoretical Issues, New York, Palgrave Macmillan, 2013.

Rorty, Richard, Philosophy and Social Hope, Londres, Penguin Books, 1999.

Simmel, Georg "Comment les formes sociales se maintiennent ", L'année sociologique, $1^{\text {ère }}$ année, 1896-1897, p. 71-109; le texte est publié en français sous la direction d'Émile Durkheim; il est reproduit dans Georg Simmel, Sociologie et épistémologie, Paris, Presses universitaires de France, 1981.

Tam, Tony, "Demarcating the Boundaries between Self and the Social: The Anatomy of Centrality in Social Networks ", Social Networks, vol. $11, n^{\circ} 4,1989$, p. 387-401.

Terenzi, Paolo, Lucia Boccacin et Ricardo Prandini Riccardo (dir.), Lessico di sociologia relazionale, Bologna, il Mulino, 2016.

Toulemont, René, L'essence de la société selon Husserl, Paris, Presses universitaires de France, 1962.

Wellman, Barry, «The Community Question: The Intimate Networks of East Yorkers ", American Journal of Sociology, vol. 84, n 5, 1979, p. 1201-1231.

Wellman, Barry, "Structure Analysis: From Method and Metaphor to Theory and Substance ", dans Barry Wellman et S. D. Berkowitz (dir.), Social Structures: A Network Approach, Cambridge, Cambridge Univesity Press, 1988, p. 19-61.

Wittgenstein, Ludwig, De la certitude, Paris, Gallimard, 1965. 